\title{
Maximum Norm Estimates in the Finite Element Method on Plane Polygonal Domains. Part 2, Refinements
}

\author{
By A. H. Schatz and L. B. Wahlbin*
}

\begin{abstract}
The finite element method is considered when applied to a model Dirichlet problem on a plane polygonal domain. Local error estimates are given for the case when the finite element partitions are refined in a systematic fashion near corners.
\end{abstract}

0. Introduction. We assume that the reader is familiar with Part 1, [2], of this paper; some notation is briefly recollected in Section 1. General references to the literature were given in the Bibliography of Part 1. Of these references, the following are particularly relevant to our present situation: Babuška [1], Babuška and Aziz [2], Babuška and Rheinboldt [4], Babuška and Rosenzweig [5], Eisenstat and Schultz [11], Thatcher [36].

Let $\Omega$ be a bounded simply connected plane polygonal domain with interior angles $0<\alpha_{1} \leqslant \cdots \leqslant \alpha_{M}<2 \pi$, and consider the Dirichlet problem

$$
\begin{aligned}
-\Delta u=f & \text { in } \Omega, \\
u=0 & \text { on } \partial \Omega,
\end{aligned}
$$

where $f$ is a given, sufficiently smooth, function.

To solve this problem numerically, let $S^{h}=S^{h}(\Omega), 0<h<1$, be a one parameter family of finite element spaces, all subspaces of $\stackrel{\circ}{H}^{1}(\Omega) \cap W_{\infty}^{1}(\Omega)$. Define the approximate solution $u_{h} \in S^{h}$ by the relation

$$
A\left(u_{h}, \chi\right)=(f, \chi) \text { for all } \chi \in S^{h} \text {, }
$$

where $A(v, w)=\int_{\Omega} \nabla v \cdot \nabla w d x$ and $(v, w)=\int_{\Omega} v w d x$.

We now describe briefly a representative result from Part 1 concerning the local rate of convergence for the finite element solution. Let $r \geqslant 2$ denote the optimal order of the parameter $h$ to which the spaces $S^{h}$ can approximate smooth functions in $L_{q}$ norms. Furthermore, let $\Omega_{j}, j=1, \ldots, M$, be the intersection of $\Omega$ with a disc of radius $R_{j}$ centered at the $j$ th vertex and such that $\Omega_{j}$ contains no other vertex, and set $\Omega_{0}=\Omega \backslash\left(\bigcup_{j=1}^{M} \bar{\Omega}_{j}\right)$. Also, put $\beta_{j}=\pi / \alpha_{j}$.

In Part 1 we showed that with $\epsilon>0$ arbitrarily small (see Part 1 , Theorem 4.1

Received March 1, 1978.

AMS (MOS) subject classifications (1970). Primary 65N30, 65N15.

* This work was supported in part by the National Science Foundation. 
for the precise hypotheses),

$$
\left\|u-u_{h}\right\|_{L_{\infty}\left(\Omega_{j}\right)} \leqslant C_{\epsilon} h^{\min \left(r, \beta_{j}, 2 \beta_{M}\right)-\epsilon}, \quad j=1, \ldots, M,
$$

and

$$
\left\|u-u_{h}\right\|_{L_{\infty}\left(\Omega_{0}\right)} \leqslant C_{\epsilon} h^{\min \left(r, 2 \beta_{M}\right)-\epsilon} .
$$

If the mesh is globally quasi-uniform, these results are essentially sharp.

It is the purpose of the present part of the paper to consider meshes that are refined in a systematic fashion near the corners, and improve upon results of Part 1 , such as the above, in this case.

We shall present the main result of this paper by means of an example problem, thus fixing our thoughts.

We consider a family of partitions $\Pi_{h}, h \rightarrow 0$, of $\Omega$ into elements, and a family of spaces $S^{h}$ of, say, continuous piecewise polynomials on such partitions. Assume, for the purposes of this introduction, that for the spaces employed an interpolant $\chi$ can be chosen such that on each element $\tau$,

$$
\|u-\chi\|_{L_{\infty}(\tau)} \leqslant C(\operatorname{diam} \tau)^{r}|u|_{W_{\infty}^{r}(\tau)} .
$$

Assume, also, that away from the corners, on $\Omega_{0}$, the diameter of some element in $\Pi_{h}$ is comparable to $h$.

For simplicity, let us fix our attention on a neighborhood $\Omega_{M}$ of the vertex of maximal angle. We wish to describe how to perform a partition of the $\Omega_{j}$ 's so as to ensure that

$$
\left\|u-u_{h}\right\|_{L_{\infty}\left(\Omega_{M}\right)} \leqslant C_{\epsilon} h^{r-\epsilon} .
$$

In general, it will be required that the diameters of the elements in $\Pi_{h}$ near the corners be less than $h$; we shall then call our meshes refined. We shall demand that the refined partitions $\Pi_{h}$ have, asymptotically, no more than $C h^{-2}$ elements, i.e., apart from the constant $C$, the same number as for an unrefined quasi-uniform mesh.

We emphasize that it is only for the purposes of this Introduction that we focus attention on obtaining optimal order estimates in a neighborhood of the vertex $v_{M}$ of maximal angle. More general situations are treated in the paper.

We first consider the question of how to refine the mesh close to the vertex $v_{M}$, and we shall seek our guidelines from the approximation estimate (0.3). Let

$$
\Omega_{M, k}=\left\{x \in \Omega: 2^{-k} R_{M} \leqslant\left|x-v_{M}\right| \leqslant 2^{-k+1} R_{M}\right\}, \quad k=1, \ldots, k_{M},
$$

where $k_{M}$ is to be chosen, and set

$$
\Omega_{M, I}=\left\{x \in \Omega:\left|x-v_{M}\right| \leqslant 2^{-k} R_{M}\right\} \text {. }
$$

Recall (Part 1, Section 1) that $\left|D^{\alpha} u(x)\right| \leqslant C\left|x-v_{M}\right|^{\beta_{M}-|\alpha|-\epsilon}$, and thus the $\Omega_{M, k}$ are regions where the bound for derivatives of $u$ is roughly constant. Employing an interpolant $\chi$ leads to, by (0.3),

$$
\|u-\chi\|_{L_{\infty}\left(\Omega_{M, k}\right)} \leqslant C h_{M, k}^{r}\left(2^{-k}\right)^{\beta M^{-r-\epsilon}},
$$


where $h_{M, k}$ denotes a local meshsize on $\Omega_{M, k}$. Desiring the right side to be $C h^{r-\epsilon}$, we see that if $\beta_{M} \geqslant r$, we may take $h_{M, k} \simeq h$ (i.e., no refinement is necessary); whereas if $\beta_{M}<r$, we should have

$$
h_{M, k} \leqslant h\left(2^{-k}\right)^{\left(1-\beta_{M} / r\right)} .
$$

An alternate way of expressing this is to say that on $\Omega_{M, k}$, if the element $\tau$ is a distance $d$ away from the corner, then

$$
\operatorname{diam} \tau \leqslant h d^{1-\beta_{M} / r} .
$$

To choose $k_{M}$, note that taking $\chi=0$ (an asymptotically optimal choice), we have

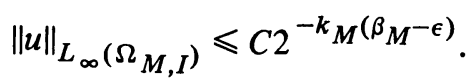

Hence, taking

$$
h_{M, I} \leqslant h^{r / \beta_{M}} \text { and } 2^{-k_{M}} \simeq h^{r / \beta} M
$$

seems reasonable. Then the innermost patch $\Omega_{M, I}$ contains a few elements of size comparable to the whole patch.

A simple calculation shows that the number of elements in a refined mesh $\Pi_{h}$ as in (0.7), (0.8) can be taken to be asymptotically comparable to $\mathrm{Ch}^{-2}$.

Our main result is that using essentially a refinement as above around the $M$ th vertex we have

$$
\left\|u-u_{h}\right\|_{L_{\infty}\left(\Omega_{M}\right)} \leqslant C h^{-\epsilon}\left\{h^{r}+\left\|u-u_{h}\right\|_{-p, \Omega}\right\}
$$

for any positive integer $p$. Thus, apart from the rightmost term in (0.9), the finite element solution mimics the pure approximation properties of $\Pi_{h}$ and $S^{h}$.

Actually, we shall need a slightly stronger refinement than the one described in (0.7), (0.8) in order to prove (0.9), viz., $h_{M, k} \leqslant h\left(2^{-k}\right)^{\left(1-\beta_{M} / r+\delta\right)}$ for some positive $\delta$. This is due to technicalities in our proof. We refer the reader to Theorem 2.1 for the exact hypotheses.

The second term on the right of (0.9) needs to be estimated. It contains the socalled "pollution effects" from other corners, and if no refinements were done at the remaining corners, the best we could say is that with $p$ large,

$$
\left\|u-u_{h}\right\|_{-p, \Omega} \leqslant C h^{\min \left(2(r-1), 2 \beta_{M-1}\right)-\epsilon} .
$$

For completeness, we shall show in Section 4 (and Appendix 1) that if certain mild refinements are performed at the remaining vertices, the term can be bounded by $C h^{r-\epsilon}$, and we thus obtain our desired estimate (0.4). Let us briefly describe the refinements necessary to alleviate the pollution effect.

If $\beta_{j} \geqslant r / 2$, no refinement is necessary at that vertex.

If $\beta_{j}<r / 2$, introduce the domains $\Omega_{j, k}, j=1, \ldots, M-1, k=k_{0, j}, \ldots, k_{j}$, and $\Omega_{j, I}$ as in (0.5), (0.6) but with $j$ replacing $M$. Choose $k_{0, j}$ such that

$$
2^{-k_{0, j}} \simeq h^{(r / 2-1) /\left(r-1-\beta_{j}\right)},
$$


and let the local meshsize $h_{j, k}$ on $\Omega_{j, k}$ satisfy

$$
h_{j, k} \leqslant h^{r / 2(r-1)}\left(2^{-k}\right)^{\left(1-\beta_{j} /(r-1)\right)}, \quad k=k_{0, j}, \ldots, k_{j} .
$$

Also, $k_{j}$ should be such that

$$
h_{j, I} \leqslant h^{r / 2 \beta_{j}} \text { and } 2^{-k_{j}} \simeq h^{r / 2 \beta_{j}} .
$$

This means that (if $r>2$ ) the refinement process can be taken to start fairly close to the corner according to $(0.10)$, and is less stringent than at the $M$ th vertex (even if $\beta_{j}$ $\left.=\beta_{M}\right)$.

The conditions $(0.10)-(0.12)$ can also be motivated from simple approximation considerations, see Section 4.

Let us remark that if an $h^{r-\epsilon}$ rate of convergence is desired only on the interior domain $\Omega_{0}$, then the weaker kind of refinement described in (0.10)-(0.12) suffices at each corner.

To elucidate the above, let us give three examples.

Example 0.1. A procedure for placing the nodes in the radial direction near $v_{M}$. Consider the problem of how to place $N+1$ nodes over $[0,1]$ so as to obtain an efficient approximation of the function $x^{\beta}\left(\beta=\beta_{M}\right)$ with piecewise polynomials of degree $r-1$. This problem was solved by Rice [1], who explicitly prescribed the location of the nodes so as to obtain a good approximation, asymptotically as $N \rightarrow \infty$. Essentially, the $N+1$ nodes $x_{i}, i=0, \ldots, N$, were taken as $x_{i}=(i / N)^{r / \beta}$.

In the two dimensional situation, one can, e.g., construct a triangular mesh near $v_{M}$ in the following fashion, Figure 1. Draw $N+1$ radial lines (including the boundaries) from $v_{M}$; along each of these mark down the $N+1$ points $x_{i}$. Then connect the $i$ th points on the successive radial lines, thus obtaining a cobweb-like set of quadrilaterals. Now triangulate those by drawing one diagonal in each. The family of triangulations obtained in this simple way will, as $N \longrightarrow \infty$, satisfy a maximum angle condition, but not a minimum angle one. In order to satisfy the latter, a more complicated construction would be necessary.

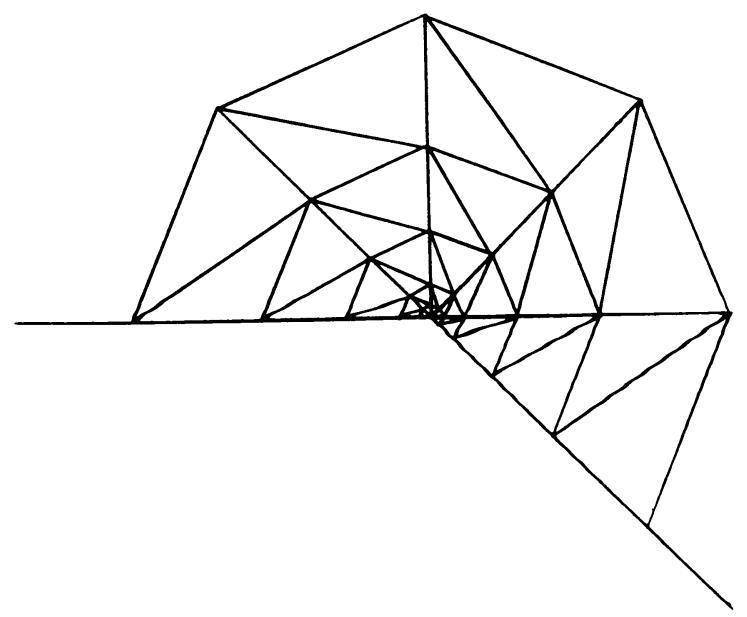

Figure 1 
Let us check that the ensuing mesh satisfies (0.7) and (0.8). Here, $h \simeq x_{N}$ $x_{N-1} \simeq(r / \beta) \cdot(1 / N)$. Clearly,

$$
h_{M, I} \leqslant C \max \left(x_{1}, \frac{x_{1}}{N}\right) \simeq C\left(\frac{1}{N}\right)^{r / \beta} \leqslant C h^{r / \beta}
$$

so that $(0.8)$ holds.

For an element $\tau$ a distance $d \simeq x_{i}$ away, we have for the meshsize $h_{\tau}$,

$$
\begin{aligned}
h_{\tau} \leqslant & C \max \left(x_{i+1}-x_{i}, \frac{x_{i+1}}{N}\right) \simeq C\left(x_{i+1}-x_{i}\right) \\
& =C\left(\left(\frac{i+1}{N}\right)^{r / \beta}-\left(\frac{i}{N}\right)^{r / \beta}\right) \\
& =\frac{C}{N}\left(x_{i}\right)^{1-\beta / r}\left[i\left\{\left(1+\frac{1}{i}\right)^{r / \beta}-1\right\}\right] \\
\leqslant & C h d^{1-\beta / r},
\end{aligned}
$$

since the quantity in square brackets is bounded independently of $i$. Thus, $(0.7)^{\prime}$ is satisfied.

Example 0.2. Piecewise quadratic elements on a triangular partition, $r=3$.

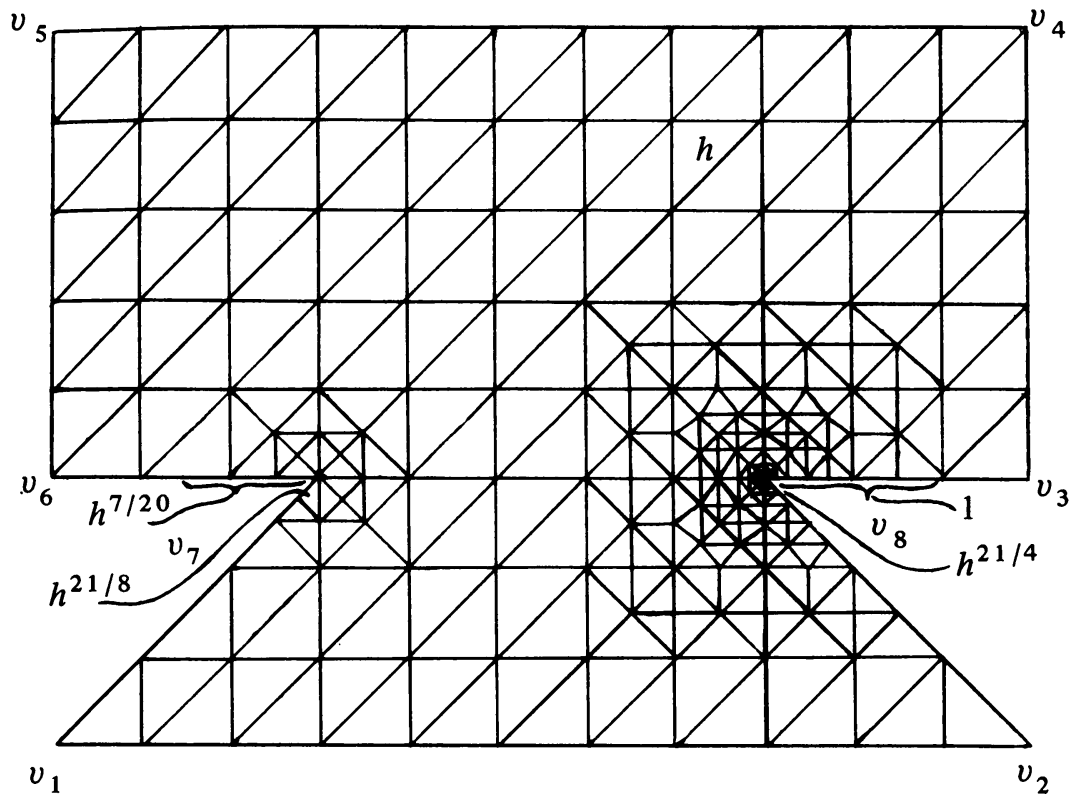

FIGURE 2

Here $\alpha_{1}=\alpha_{2}=\pi / 4, \alpha_{3}=\cdots=\alpha_{6}=\pi / 2, \alpha_{7}=\alpha_{8}=7 \pi / 4$. We seek a sequence of meshes with local meshsize $h$ in the interior such that $O\left(h^{3}\right)$ convergence will occur at $v_{8}$. We find that no refinement is necessary at the vertices $v_{1} \cdots v_{6}$. At $v_{7}$, a mild refinement according to (0.10)-(0.12) is required, and in Figure 2 we have displayed $2^{-k} 0,7=h^{7 / 20}$, i.e. the distance where the refinement starts, and $h_{7, I}=h^{21 / 8}$, the smallest meshsize employed right at the vertex $v_{7}$. Finally, at $v_{8}$ we refine according 
to (0.7) and (0.8) starting a unit distance away from the corner; again we have displayed in the figure the innermost meshsize, $h^{21 / 4}$.

Example 0.3. Piecewise bilinear functions on a rectangular partition, $r=2$.

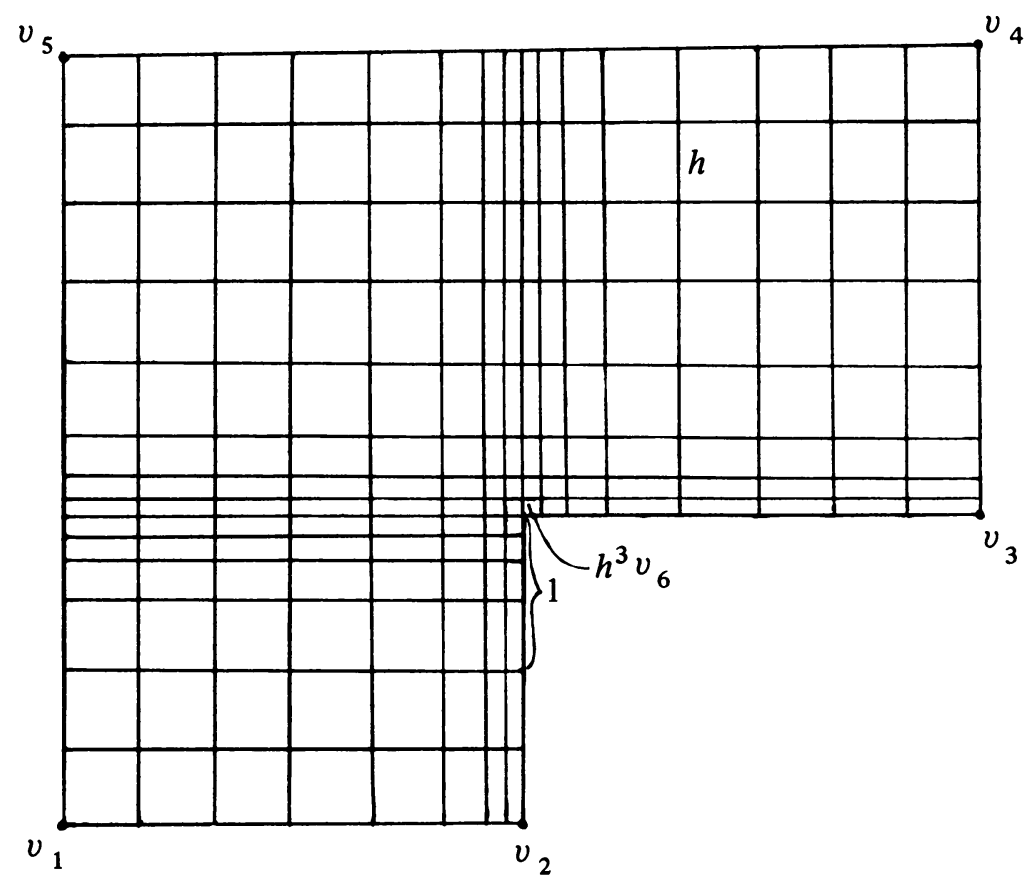

FIGURE 3

Here $\alpha_{1}=\cdots=\alpha_{5}=\pi / 2, \alpha_{6}=3 \pi / 2$. To obtain $h^{2-\epsilon}$ convergence at $v_{6}$, no refinement is necessary at the other corners, whereas at $v_{6}$ one needs to refine so that the innermost meshsize is $\simeq h^{3}$.

Note that if the mesh is built up in a tensor-product fashion as indicated in Figure 3 , then it will not be locally quasi-uniform. In fact, the "thinnest" elements are found away from the comers. Our theory still applies in this situation.

Incidentally, in this example the convergence rate will be $h^{2-\epsilon}$ on the whole of $\Omega$, without any refinements at the vertices $v_{1} \cdots v_{5}$.

Remark. If the meshsize is roughly halved on each adjoining $\Omega_{M, k}$, i.e., $h_{M, k}$ $\simeq h 2^{-k}$, then the corresponding refinement satisfies (0.7). However, in this case the number of elements will be asymptotically comparable to $C h^{-2} \log 1 / h$.

To attain an $h^{r}$ rate of convergence one may sometimes be led to rather small meshsizes at the vertices; cf. Example 0.2. In Section 3 we shall give a corresponding analysis when an asymptotic convergence rate of $h^{s}, s<r$, is desired. As in (0.10) above, we find that the refinement may then be started closer to the corner than the unit distance demanded when optimal convergence is sought. In this case of suboptimal refinements we shall also consider briefly (in two examples, Section 5) the determination of the rate of convergence as a function of the distance to the vertex, and the calculation of stress intensity factors. Similar investigations are given in Part 1, Section 6, where the results are sharp for meshes where all the elements are roughly of size $h$. 
In this Introduction we have considered refinements based on approximation in the maximum norm. One can also base the refinement procedure on approximation properties in other norms; our analysis still applies to give maximum norm estimates. As an example, if one uses the energy norm and aims for optimal $h^{r-1}$ convergence in that norm, one obtains a refinement which is suboptimal in our sense, with $s=r-1$.

Finally, let us emphasize that the local estimate (0.9) applies to other problems than the Dirichlet problem discussed here. For example, outside of $\Omega_{M}$ the boundary does not have to be polygonal, nor do the boundary conditions have to be of Dirichlet type. The second term on the right has to be estimated in each case.

Outline of the Paper. In Section 1 we recall some notation. The main result of the paper is stated in Section 2 (its proof is given in Section 6). There we describe the refinements necessary to obtain (0.9), i.e., optimal order $O\left(h^{r}\right)$ convergence at a corner, not counting pollution. In Section 3 the same question is considered when suboptimal $O\left(h^{s}\right)$ order, $s<r$, convergence is desired-again not heeding pollution. The pollution effect is dealt with in Section 4. Section 5 contains examples of suboptimal refinements at one corner where an overall refinement is made to give optimal convergence in the interior. The question of the dependence of the rate of convergence at a point on its distance to the vertex is investigated.

1. Notation. We first recall relevant terminology from Part 1, and then introduce some new notation.

For $D_{1} \subseteq D \subseteq \Omega$, define

$$
\operatorname{dist}_{\Varangle}\left(D_{1}, D\right)=\inf _{x \in \partial D_{1} \backslash\left(\partial D_{1} \cap \partial \Omega\right)} \operatorname{dist}(x, \partial D \backslash(\partial D \cap \partial \Omega)),
$$

and let $D_{1} \Varangle D$ mean that $D_{1} \subseteq D$ with $\operatorname{dist}_{\Varangle}\left(D_{1}, D\right)>0$.

For $D \subseteq \Omega$ we set

$$
\stackrel{\leftrightarrow}{H}^{p}(D)=\left\{v \in H^{p}(D): v=0 \text { on } \partial D \cap \partial \Omega\right\}
$$

and

$$
\not{H}^{p}(D)=\left\{v \in H^{p}(D): v \equiv 0 \text { in a neighborhood of } \partial D \backslash(\partial D \cap \partial \Omega)\right\} \text {. }
$$

The spaces $\stackrel{<}{C}^{\infty}(D), \stackrel{C}{C}^{\infty}(D)$ and $\stackrel{\$}{h}^{h}(D)$ are defined in a similar fashion.

For $p<0$, set

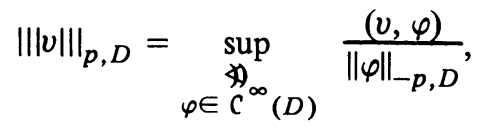

$$
\begin{aligned}
& \|v\|_{p, D}=\sup _{\varphi \in \mathcal{C}^{\infty}(D) \cap C^{\infty}(D)} \frac{(v, \varphi)}{\|\varphi\|_{-p, D}}
\end{aligned}
$$

where $\|\cdot\|_{-p, D}$ is the norm in $H^{-p}(D)$. Note that $\mathrm{C}^{\infty}(\Omega) \equiv C^{\infty}(\Omega)$.

We set $\beta_{j}=\pi / \alpha_{j}, j=1, \ldots, M$.

Let

$$
\Omega_{j}=\left\{x \in \bar{\Omega}:\left|x-v_{j}\right|<R_{j}\right\}, \quad j=1, \ldots, M,
$$


for some $R_{j}$ such that $\bar{\Omega}_{j}$ contains no other vertex than $v_{j}$. Also, for some $\widetilde{R}_{j}<R_{j}$,

$$
\widetilde{\Omega}_{j}=\left\{x \in \bar{\Omega}:\left|x-v_{j}\right|<\widetilde{R}_{j}\right\} .
$$

Relative to $\Omega_{j}$, we introduce the following domains, some of which were already described in the Introduction.

$$
\begin{gathered}
\Omega_{j, k}=\left\{x \in \bar{\Omega}: 2^{-k} R_{j} \leqslant\left|x-v_{j}\right| \leqslant 2^{-k+1} R_{j}\right\}, \\
\Omega_{j, k}^{l}=\left\{\Omega_{k+l} \cup \cdots \cup \Omega_{k-l}\right\} \cap \Omega_{j}, \quad l=0,1,2, \ldots .
\end{gathered}
$$

Given an integer $k_{j}$ we define relative to that integer (but suppress that dependence in the notation)

$$
\begin{gathered}
\Omega_{j, I}=\left\{x \in \Omega:\left|x-v_{M}\right| \leqslant 2^{-k_{j}} R_{j}\right\}, \\
\Omega_{j, I}^{l}=\left\{\Omega_{j, I} \cup \Omega_{k_{j}-1} \cup \cdots \cup \Omega_{k_{j}-l}\right\} \cap \Omega_{j}, \quad l=0,1,2, \ldots .
\end{gathered}
$$

Lastly, as in Part 1, we make the convention that $\epsilon$ is an arbitrarily small positive number, not necessarily the same at each occurrence. Constants $C$, which are also subject to change without notice, may depend on $\epsilon$.

2. Optimal Order Refinements Near a Corner. In this section we fix our attention in a neighborhood of a certain vertex $v_{j}$ of interior angle $\alpha_{j}$. Set $\beta_{j}=\pi / \alpha_{j}$, and let $\widetilde{\Omega}_{j} \Omega_{j}$ be defined as in (1.1), (1.2).

Loosely speaking, the aim of this section is the following: Assume that locally the class of spaces $S^{h}$ employed is capable of order $h_{\text {loc }}^{r}$ approximation in the maximum norm for smooth functions, where $h_{10 c}$ is a "local" meshsize, cf. (0.3). We want to describe a class of refinements on $\Omega_{j}$ that leads to an error estimate of the form

$$
\left\|u-u_{h}\right\|_{L_{\infty}\left(\tilde{\Omega}_{j}\right)} \leqslant C_{\epsilon} h^{-\epsilon}\left\{h^{r}+\left\|u-u_{h}\right\| L_{-p, \Omega_{j}}\right\} .
$$

Note that if $\beta_{j} \geqslant r$, this estimate follows from the results of Part 1 using only an "unrefined" mesh.

The assumptions needed to obtain (2.1) will now be described, in a slightly longwinded fashion. We shall refer to the whole of them as AA.2 $2_{j}(r)$.

AA. $2_{j}(r)$. Let there be given numbers $r, \beta_{j}$ and $\gamma$ with $r \geqslant 2$ integer, $1 / 2<\beta_{j}, \gamma$ $\geqslant 1$. Our assumptions are divided into two parts, (i) and (ii) below.

(i) The spaces $S^{h}\left(\Omega_{j}\right)$ satisfy the assumptions A.1-A.4 of Part 1.

Remarks. Recall that A.1 went as follows:

There exist constants $k_{0}$ and $C_{1}$ such that the following holds. Let $D_{1} \ggg D$ with dist $_{\Varangle}\left(D_{1}, D\right) \geqslant k_{0} h$. Then for each $v \in W_{\infty}^{r}(D)$ and vanishing on $\partial D \cap \partial \Omega$, there exists a $\chi \in S^{h}(D)$ such that

$$
\|v-\chi\|_{L_{\infty}\left(D_{1}\right)}+h\|v-\chi\|_{W_{\infty}^{1}\left(D_{1}\right)} \leqslant C_{1} h^{r}|v|_{W_{\infty}^{r}(D)} .
$$

Furthermore, if $v \in H^{1}\left(D_{1}\right)$, then $\chi \in S^{h}(D)$.

The assumptions A.2-A.4 shall never be used explicitly in this paper, and hence 
we do not recall them. They are needed so that we can quote results from Part 1. These assumptions were, respectively, concerned with "superapproximation", weak inverse estimates, and the behavior of the finite element spaces under homotheties.

In the case of $\beta_{j}<r$ we make additional hypotheses that reflect the fact that the mesh is then refined near $v_{j}$.

(ii) If $1 / 2<\beta_{j}<r$, let $\mu_{j}$ be a number with

$$
1-\beta_{j} / r<\mu_{j} \leqslant 1
$$

such that (in addition to (i)) the following holds, cf. (1.4), (1.6) for notation. Let

$$
k_{j}=\left[\frac{r-\beta_{j}}{\mu_{j} \beta_{j}} \ln _{2} \frac{1}{h}\right] .
$$

On each $\Omega_{j, k}^{1}, k=1, \ldots, k_{j}, S^{h}\left(\Omega_{j, k}^{1}\right)$ satisfies the assumptions A.2-A.4 of Part 1 with $h$ replaced by a local meshsize $h_{j, k}$,

$$
h^{\gamma} \leqslant h_{j, k} \leqslant h 2^{-k \mu_{j}}
$$

On $\Omega_{j, I}^{1}, S^{h}\left(\Omega_{j, k}^{1}\right)$ satisfies A.2-A.4 of Part 1 with $h$ replaced by $h_{j, I}$,

$$
h^{\gamma} \leqslant h_{j, I} \leqslant h^{r / \beta_{j}} \text {. }
$$

We also need an approximation assumption corresponding to A.1, with respect to local meshsizes. There exist constants $k_{0}, C_{1}$ such that for each function $v \in$ $W_{\infty}^{r}\left(\Omega_{j}\right)$ vanishing on $\partial \Omega$, there exists $\chi$ in $S^{h}\left(\Omega_{j}\right)$ such that for $D_{1} \ \Omega_{j, k}^{1}$ (or $\left.\Omega_{j, I}^{1}\right)$ with $\operatorname{dist}_{\Varangle}\left(D_{1}, D\right) \geqslant k_{0} h_{j, k}\left(\right.$ or $\left.k_{0} h_{j, I}\right)$,

$$
\|v-\chi\|_{L_{\infty}\left(D_{1}\right)}+h_{j, k}\|v-\chi\|_{W_{\infty}^{1}\left(D_{1}\right)} \leqslant C_{1} h_{j, k}^{r}|v|_{W_{\infty}^{r}(D)}
$$

(or $h_{j, I}$ replacing $h_{j, k}$ ). If furthermore $v \in H^{1}\left(D_{1}\right)$, then $\chi \in S^{\sharp}(D)$.

This ends the description of AA. $2_{j}(r)$.

In particular, the assumptions in (ii) make it possible to quote local results on $\Omega_{j, k}$ from Part 1 with $h_{j, k}$ replacing $h$.

Loosely speaking, the last part of (ii) says that A.1 holds on each $\Omega_{j, k}\left(\Omega_{j, I}\right)$ with $h$ replaced by a local meshsize satisfying (2.4) (or (2.5)). This approximation assumption implies other results on approximation with respect to other norms, and for nonsmooth functions. These results will be listed at the appropriate place in our development, when needed, and brief indications of their proofs given. Generally the proofs, or very similar ones, were given in Part 1.

We note that in the assumptions, $\mu_{j}$ is assumed to be strictly greater than 1 $\beta_{j} / r$; this is due to technicalities in our proof. Thus, e.g., our Example 0.1 has to be changed slightly so that $x_{i}=(i / N)^{1-\beta / r+\delta}$, some $\delta>0$, in order to fit that part of our hypotheses. If a family of meshes satisfies AA. $2_{j}(r)$ with some $\mu_{j}>1-\beta_{j} / r$, then it does so for any $\tilde{\mu}_{j}$ with $1-\beta_{j} / r<\tilde{\mu}_{j}<\mu_{j}$. Loosely speaking, the larger the $\mu_{j}$ that the mesh allows, the more "over-refined" it is.

The innermost domain $\Omega_{j, I}$ may be thought of as the part where a meshsize $h^{r / \beta_{j}}$ 
prevails. Note that $\Omega_{j, I}$ depends on $\mu_{j}$; this will be convenient in the proofs. For $\mu_{j}$ close to $1-\beta_{j} / r$, the innermost part may contain only a few elements, whereas for $\mu_{j}$ $=1$ it contains on the order of $\mathrm{Ch}^{-2}$ elements.

Our hypotheses are satisfied for example by:

(1) continuous piecewise polynomials of degree $r-1$ on suitably refined triangulations that satisfy a minimum angle condition;

(2) piecewise linear functions $(r=2)$ on suitably refined triangulations that satisfy a maximum angle condition;

(3) piecewise bilinear functions on a suitable tensor product mesh $(r=2)$; this will, in general, contain "thin" rectangles, cf. Example 0.3.

For verification of all other hypotheses, given that $(2.4),(2.5)$ hold, in the cases listed above, we refer to Part 1.

We can now state our main result.

THEOREM 2.1. Let $j$ be fixed and assume that the family of spaces $S^{h}\left(\Omega_{j}\right), 0<$ $h<1$, satisfies AA.2 ${ }_{j}(r)$. Let $\epsilon>0$ and an integer $p \geqslant 0$ be given.

Assume that

$$
A\left(u-u_{h}, \chi\right)=0 \quad \text { for all } \chi \in S^{h}\left(\Omega_{j}\right) .
$$

There exists a constant $C$ such that for $h$ sufficiently small,

$$
\left\|u-u_{h}\right\|_{L_{\infty}\left(\tilde{\Omega}_{j}\right)} \leqslant C h^{-\epsilon}\left\{h^{r}+\left\|u-u_{h}\right\|_{-p, \Omega_{j}}\right\} .
$$

The proof of Theorem 2.1 will be given in Section 6 .

3. Suboptimal Order Refinements Near a Corner. In this section we shall consider the following question: Starting with an unrefined mesh of size $h$ and capable of $h^{r}$ approximation for smooth functions, and given a number $s, 0<s<r$, how should one refine near the $j$ th corner to obtain the estimate

$$
\left\|u-u_{h}\right\|_{L_{\infty}\left(\widetilde{\Omega}_{j}\right)} \leqslant C h^{-\epsilon}\left\{h^{s}+\left\|u-u_{h}\right\| \|_{-p, \Omega_{j}}\right\} ?
$$

We note that (3.1) would follow from Theorem 2.1 if AA. $2_{j}(r)$ holds with $h$ replaced by $h^{s / r}$. However, now we have assumed that a unit distance away, the meshsize is to be of order $h$, which is obviously less than $h^{s / r}$. We shall show in this situation that we need only start to refine the mesh closer than a unit distance away from the corner. Exactly how this is done can be motivated from approximation theory, just as AA.2 ${ }_{j}(r)$ was motivated in the Introduction. We leave this motivation to the reader and proceed to list our formal assumptions.

AA. $3_{j}(r, s)$. Let there be given numbers $r, \beta_{j}, \gamma$ and $s$ with $r \geqslant 2$ an integer, $1 / 2$ $<\beta_{j}, \gamma \geqslant 1,0<s \leqslant r$.

(i) The spaces $S^{h}\left(\Omega_{j}\right)$ satisfy A.1-A.4 of Part 1 .

(ii) If $1 / 2<\beta_{j}<s$, let $\mu_{j}$ be a number with $1-\beta_{j} / r<\mu_{j} \leqslant 1$ such that (in addition to (i)) the following holds. Set

$$
k_{0, j}=\left[\frac{r-s}{r \mu_{j}} \ln _{2} \frac{1}{h}\right], \quad k_{j}=\left[\frac{s}{\mu_{j}}\left(\frac{1}{\beta_{j}}-\frac{1}{r}\right) \ln _{2} \frac{1}{h}\right] .
$$


The rest of the assumption now reads like AA. $2_{j}(r)$, with the following change in the local meshsizes:

$$
\begin{aligned}
& h^{\gamma} \leqslant h_{j, k} \leqslant h, \quad k=1, \ldots, k_{0, j}-1, \\
& h^{\gamma} \leqslant h_{j, k} \leqslant h^{s / r_{2}}{ }^{-k \mu_{j}}, \quad k=k_{0, j}, \ldots, k_{j}, \\
& h^{\gamma} \leqslant h_{j, I} \leqslant h^{s / \beta_{j}} .
\end{aligned}
$$

The observation that AA.2 ${ }_{j}(r)$ is satisfied with $h$ replaced by $h^{s / r}$ leads immediately to the following corollary to Theorem 2.1 .

COROLlARY 3.1. Let $j$ be fixed and assume that with $0<s \leqslant r$, the family of spaces $S^{h}\left(\Omega_{j}\right), 0<h<1$, satisfies AA. $3_{j}(r, s)$. Let $\epsilon>0$ and an integer $p \geqslant 0$ be given, and let (2.7) hold. There exists a constant $C$ such that

$$
\left\|u-u_{h}\right\|_{L_{\infty}\left(\tilde{\Omega}_{j}\right)} \leqslant C h^{-\epsilon}\left\{h^{s}+\left\|u-u_{h}\right\|_{-p, \Omega_{j}}\right\} .
$$

4. Error Estimates Near a Corner, and Global Estimates. Fix a vertex $v_{j}$ and a number $s, 0<s \leqslant r$. Assume that

$$
\left\|u-u_{h}\right\|_{L_{\infty}}\left(\widetilde{\Omega}_{j}\right) \leqslant C h^{-\epsilon}\left\{h^{s}+\left\|u-u_{h}\right\|_{-p, \Omega}\right\},
$$

cf. Corollary 3.1. We now ask whether we can achieve

$$
\left\|u-u_{h}\right\|_{L_{\infty}\left(\tilde{\Omega}_{j}\right)} \leqslant C h^{s-\epsilon} .
$$

For this, the second term on the right of (4.1) needs to be estimated-this term contains the pollution effects from other corners.

Let us choose $p=r-2$ and ask for an estimate

$$
\left\|u-u_{h}\right\|_{2-r, \Omega} \leqslant C h^{s-\epsilon} .
$$

We shall describe the kind of refinements necessary at the corners in order to achieve (4.3). Roughly speaking, we shall refine at the other corners so that globally $\left\|u-u_{h}\right\|_{1, \Omega} \leqslant C h^{s / 2-\epsilon}$, and (4.3) then follows by a standard duality argument. Again the description of the meshes will be motivated by somewhat imprecise approximation considerations. The full proof will be given in Appendix 1 .

We have

$$
\left\|u-u_{h}\right\|_{2-r, \Omega}=\sup _{\substack{g \in C^{\infty}(\Omega) \\\|g\|_{r-2, \Omega}=1}}\left(u-u_{h}, g\right) .
$$

For each fixed $g$, let $v$ be the solution of the following Dirichlet problem:

$$
\begin{aligned}
-\Delta v=g & \text { in } \Omega, \\
v=0 & \text { on } \partial \Omega .
\end{aligned}
$$

Then for any $\chi \in S^{h}(\Omega)$,

$$
\left|\left(u-u_{h}, g\right)\right|=\left|A\left(u-u_{h}, v\right)\right|=\left|A\left(u-u_{h}, v-\chi\right)\right| \leqslant\left\|u-u_{h}\right\|_{1, \Omega}\|v-\chi\|_{1, \Omega} .
$$

Note that $\left\|u-u_{h}\right\|_{1, \Omega} \leqslant C\|u-\psi\|_{1, \Omega}$ for any $\psi \in S^{h}(\Omega)$. 
The refinements we seek achieve

$$
\inf _{\chi \in S^{h}(\Omega)}\|v-\chi\|_{1, \Omega} \leqslant C h^{s / 2-\epsilon}\|g\|_{r-2, \Omega}
$$

and by the above, (4.3) would follow. Instead of showing (4.5), let us motivate how an estimate of the form

$$
\|v-\chi\|_{1, \Omega} \leqslant C(v) h^{s / 2-\epsilon}, \quad v \text { solution of (4.4), }
$$

may be achieved. The full details for (4.5) will be given in Appendix 1.

We have

$$
\|v-\chi\|_{1, \Omega} \leqslant\|v-\chi\|_{1, \Omega_{0}}+\sum_{j=1}^{M}\|v-\chi\|_{1, \Omega_{j}} .
$$

On $\Omega_{0}$, the function $v$ is smooth, and we may assume that without any refinement,

$$
\|v-\chi\|_{1, \Omega_{0}} \leqslant C h^{r-1} \text {. }
$$

Consider next a fixed $\Omega_{j}$. Then, cf. (1.1), (1.3) and (1.5) for notation,

$$
\|v-\chi\|_{1, \Omega_{j}} \leqslant\|v-\chi\|_{1, \Omega_{j, I}}+\sum_{k=1}^{k_{j}}\|v-\chi\|_{1, \Omega_{j, k}} .
$$

On the innermost domain $\Omega_{j, I}$, a meshlength $h_{j, I}$ prevails, and we have, using properties of $v$, cf. Part 1, (1.10)-(1.11),

$$
\|v-\chi\|_{1, \Omega_{j, I}} \leqslant C h_{j, I}^{\beta}-\epsilon
$$

On the $\Omega_{j, k}$ with meshsize $h_{j, k}$, using again (1.10), (1.11) of Part 1,

$$
\|v-\chi\|_{1, \Omega_{j, k}} \leqslant C h_{j, k}^{r-1}\|v\|_{r, \Omega}^{1} \leqslant C h_{j, k}^{r-1}\left(2^{-k}\right)^{\beta,-r+1-\epsilon} .
$$

To make the right-hand sides of (4.7), (4.8) less than $h^{s / 2-\epsilon}$, one needs

$$
\begin{gathered}
h_{j, I} \leqslant h^{s / 2 \beta_{j}}, \\
h_{j, k} \leqslant h^{s / 2(r-1)}\left(2^{-k}\right)^{\left(1-\beta_{j} /(r-1)\right)} .
\end{gathered}
$$

The process of refinement to achieve (4.6) can be described as follows. If $\beta_{j} \geqslant s / 2$, no refinement is necessary.

If $\beta_{j}<s / 2$, start refining on $\Omega_{k_{0, j}}$ when the right-hand side of (4.10) is less than $h$ (so that $(4.10)$ is not satisfied by the unrefined mesh), and continue gradually until a mesh of size $h^{s / 2 \beta_{j}}$, cf. (4.9), is reached; use that meshsize on the innermost patch $\Omega_{j, I}$.

To be more precise let us demand:

AA.4 $(r, s)$. Let there be given numbers $r, \gamma$ and $s$ with $r \geqslant 2$ an integer, $\gamma \geqslant 1$, $0<s \leqslant r$.

(i) $S^{h}(\Omega)$ satisfies A.1-A.4 of Part 1 .

(ii) $)_{j}$ If $\beta_{j}<s / 2$, let $\bar{\mu}_{j}$ be a number with $1-\beta_{j} /(r-1) \leqslant \bar{\mu}_{j} \leqslant 1$ such that the 
following holds. Set

$$
\begin{gathered}
k_{0, j}=\left[\left(1-\frac{s}{2(r-1)}\right) \frac{1}{\bar{\mu}_{j}} \ln _{2} \frac{1}{h}\right], \\
k_{j}=\left[\frac{s}{2 \bar{\mu}_{j}}\left(\frac{1}{\beta_{j}}-\frac{1}{r-1}\right) \ln _{2} \frac{1}{h}\right] .
\end{gathered}
$$

The rest of the assumption now reads like AA. $2_{j}(r)$, with the following change in the local meshsizes:

$$
\begin{aligned}
& h^{\gamma} \leqslant h_{j, k} \leqslant h, \quad k=1, \ldots, k_{0, j}-1, \\
& h^{\gamma} \leqslant h_{j, k} \leqslant h^{s / 2(r-1)}\left(2^{-k}\right)^{\bar{\mu}_{j}}, \quad k=k_{0, j}, \ldots, k_{j}, \\
& h^{\gamma} \leqslant h_{j, I} \leqslant h^{s / 2 \beta_{j}} .
\end{aligned}
$$

Remark 4.1. It is easily established that if $\mathrm{AA}_{. j}(r, s)$ holds (for some $\mu_{j}$ ), then the part (ii) ${ }_{j}$ of AA.4 $(r, s)$ is also satisfied (with suitable $\bar{\mu}_{j}$ ).

We shall prove the following in Appendix 1.

THEOREM 4.1. Let $0<s \leqslant r$, and assume that the family of spaces $S^{h}(\Omega), 0<$ $h<1$, satisfies AA.4( $r, s)$. Let $\epsilon>0$ be given. There exists a constant $C$ such that if $u$ and $u_{h} \in S^{h}(\Omega)$ satisfy (0.1) and (0.2), then

$$
\|\| u-u_{h} \|_{2-r, \Omega} \leqslant C h^{s-\epsilon} .
$$

Combining Corollary 3.1 and Theorem 4.1, we have:

COROLlary 4.1. Let $j$ be fixed and assume that with $0<s \leqslant r$, the family of spaces $S^{h}(\Omega), 0<h<1$, satisfies AA. $3_{j}(r, s)$ around the vertex $v_{j}$ and the global condition AA.4(r, s). Let $\epsilon>0$ be given. There exists a constant $C$ such that for $h$ sufficiently small,

$$
\left\|u-u_{h}\right\|_{L_{\infty}\left(\tilde{\Omega}_{j}\right)} \leqslant C h^{s-\epsilon}
$$

Finally, we have the following global result, cf. Remark 4.1 .

COROLlARY 4.2. Let $0<s \leqslant r$, and assume that the family $S^{h}(\Omega), 0<h<1$, satisfies AA.3. $(r, s)$ for each $j=1, \ldots, M$, and furthermore satisfies A.1-A.4 of Part 1. Let $\epsilon>0$ be given. There exists a constant $C$ such that for $h$ sufficiently small,

$$
\left\|u-u_{h}\right\|_{L_{\infty}(\Omega)} \leqslant C h^{s-\epsilon} .
$$

In particular, the above corollaries hold for $s=r$; in this case the condition AA. $3_{j}(r, r)$ is the same as AA. $2_{j}(r)$.

5. More on Suboptimal Refinements. Let $\beta_{M}\langle 1, r\rangle s$, and consider the situation when AA. $3_{M}(r, s)$ and AA.4 $(r, r)$ hold; i.e., the mesh is globally defined so that the error is $h^{r}$ in the interior of $\Omega$, and $h^{s}$ close to $v_{M}$. One may then surmise that the rate of convergence at a point near $v_{M}$ depends in some fashion on its distance to $v_{M}$. We shall show such is the case in two examples, and also consider briefly the question of calculation of stress intensity factors; cf. Part 1, Section 6 . The 
techniques used in these two examples can be applied to analyze many other situations. It may be laborious, though, to obtain sharp estimates in a particular case.

We start with an example which is easy to analyze with our present tools.

Example 5.1. $r=4, s=3, \beta_{M}=2 / 3$. Let us plot how the local meshlength $h_{\text {loc }}$ near $v_{M}$ depends on the distance $d$ from $v_{M}$ (for simplicity in plotting we demand sharpness in the right-hand sides in AA. ${ }_{M}(4.3)$ and AA.4(4.4) with "lowest" $\mu, \bar{\mu}$ ).

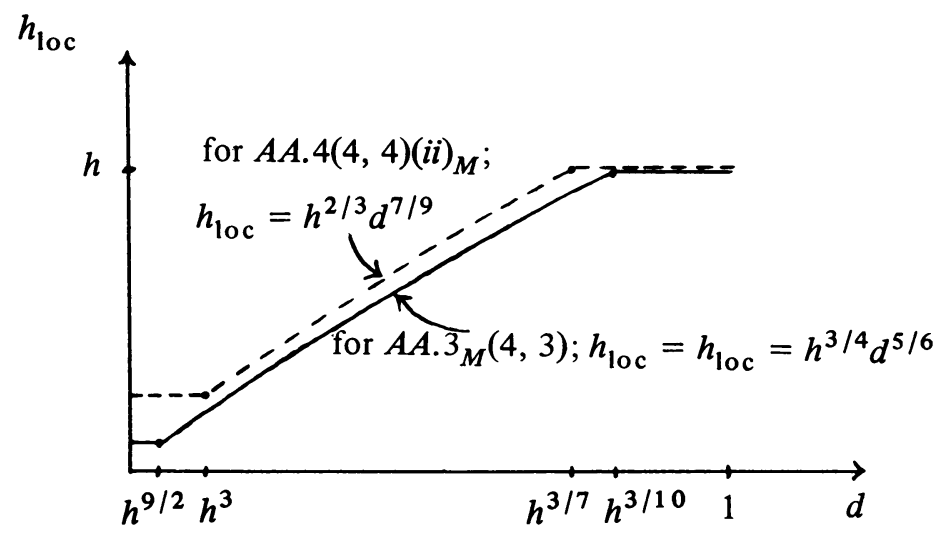

FIGURE 4

We see here that the refinement according to AA. $3_{M}(4,3)$ suffices to satisfy the relevant part of AA.4 $(4,4)$ at $v_{M}$.

We shall first show that in this example, with $d_{l}=2^{-l}$,

$$
\left\|u-u_{h}\right\|_{L_{\infty}\left(\Omega_{M, l}\right)} \leqslant \begin{cases}C h^{3-\epsilon}, & d_{l} \leqslant h^{3 / 10}, \\ C h^{4-\epsilon} d_{l}^{-10 / 3}, & h^{3 / 10}<d_{l} \leqslant R_{M} .\end{cases}
$$

For $d \leqslant h^{3 / 10}$, the refinement is done as to insure an $h^{3}$ estimate. For $d>$ $h^{3 / 10}$, we apply Theorem 3.2 of Part 1 which gives on $\Omega_{M, l}$, where $d_{l}>h^{3 / 10}$ and $h_{l} \equiv h$

$$
\begin{aligned}
& \left\|u-u_{h}\right\|_{L_{\infty}\left(\Omega_{M, l}\right)} \\
& \quad \leqslant C h^{-\epsilon}\left\{h\|u-\chi\|_{w_{\infty}^{1}\left(\Omega_{M, l}^{1}\right)}+\|u-\chi\|_{L_{\infty}\left(\Omega_{M, l}^{1}\right)}+d_{l}^{-3}\|\| u-u_{h} \|_{-2, \Omega}^{1}\right\}
\end{aligned}
$$

for any $\chi \in S^{h}(\Omega)$. The condition AA.4(4,4) was done so that (Theorem 4.1)

$$
\left\|u-u_{h}\right\|_{-2, \Omega}^{1} \leqslant, l=u_{h} \|_{-2, \Omega} \leqslant C h^{4-\epsilon} ;
$$

and hence, we obtain using approximation and the behavior of $u$,

$$
\left\|u-u_{h}\right\|_{L_{\infty}\left(\Omega_{M, l}\right)} \leqslant C\left(h^{4-\epsilon} d_{l}^{2 / 3-4}+h^{4-\epsilon} d_{l}^{-3}\right) .
$$

The first term here, coming from approximation theory, dominates and so (5.1) obtains.

In particular, it follows from (5.1), cf. Part 1 , Section 5, that for $h$ sufficiently small the maximal error occurs for $d \leqslant h^{3 / 10}$. 
Consider now the calculation of the "stress intensity factor" $k_{M}$, cf. Part 1, Section 6. Let (using polar coordinates)

$$
k_{M}(d, h)=\frac{u_{h}\left(d, \theta_{0}\right)}{d^{2 / 3} \sin \left(\beta_{M} \theta_{0}\right)} .
$$

Then, from Part 1, Section 6, and the above

$$
\begin{aligned}
\left|k_{M}-k_{M}(d, h)\right| & \leqslant C\left(\frac{\left|\left(u-u_{h}\right)\left(d, \theta_{0}\right)\right|}{d^{2 / 3}}+d^{2 / 3}\right) \\
& \leqslant \begin{cases}h^{3-\epsilon} d^{-2 / 3-\epsilon}+d^{2 / 3}, & d \leqslant h^{3 / 10}, \\
h^{4-\epsilon} d^{-4-\epsilon}+d^{2 / 3}, & h^{3 / 10} \leqslant d \leqslant R_{M} .\end{cases}
\end{aligned}
$$

It is easily seen that the best that can be gotten from this is the estimate

$$
\left|k_{M}-k_{M}\left(h^{9 / 4}, h\right)\right| \leqslant C h^{3 / 2-\epsilon} \text {. }
$$

Example 5.2. $r=2, s=1, \beta_{M}<1$. Again we plot the local meshlength near $v_{M}$ as a function of the distance (taking equality and lowest $\mu, \bar{\mu}$ in AA.3 and AA.4).

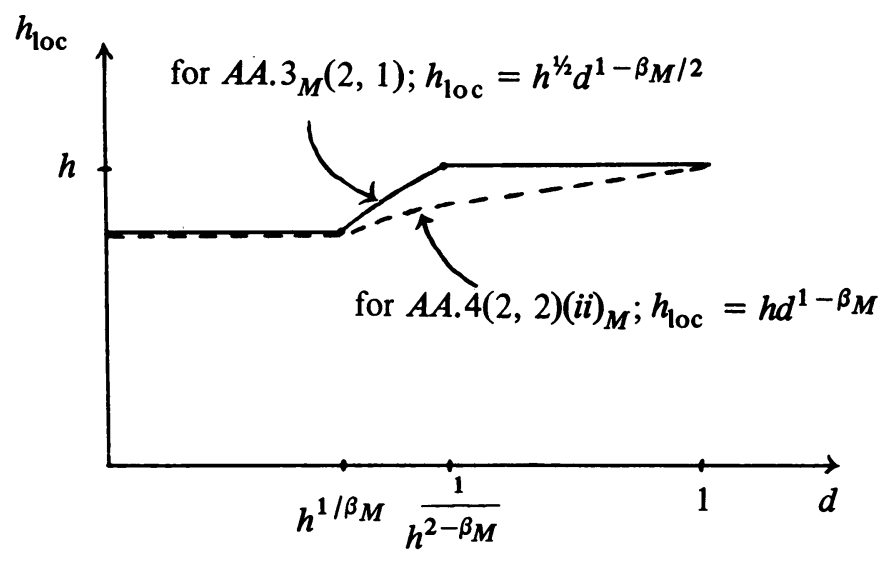

FIGURE 5

In contrast to Example 5.1, here it is the refinement demanded by AA.4(2,2) that dominates around $v_{M}$. We shall show that

$$
\left\|u-u_{h}\right\|_{L_{\infty}\left(\Omega_{M, l}\right)} \leqslant \begin{cases}C h^{1-\epsilon}, & d_{l} \leqslant h^{1 / \beta} M \\ C h^{2-\epsilon} d_{l}^{-\beta_{M}-\epsilon}, & h^{-\beta_{M} \leqslant d_{l} \leqslant R_{M} .}\end{cases}
$$

For $d \leqslant h^{1 / \beta_{M}}$ the refinements coincide; and thus, we cannot expect better than

$$
\left\|u-u_{h}\right\|_{L_{\infty}\left(\Omega_{M, I}\right)} \leqslant C h^{1-\epsilon} \text {. }
$$


For $d>h^{1 / \beta} M$, we again apply Theorem 3.2 of Part 1 to obtain $\left\|u-u_{h}\right\|_{L_{\infty}\left(\Omega_{M, l}\right)}$

$$
\leqslant C h^{-\epsilon}\left\{h_{l}\|u-\chi\|_{W_{\infty}^{1}\left(\Omega M_{M, l}\right)}+\left\|u-u_{h}\right\|_{L_{\infty}\left(\Omega \frac{1}{M, l}\right)}+d_{l}^{-1}\left\|u-u_{h}\right\|_{0, \Omega \frac{1}{M, l}}\right\},
$$

for any $\chi \in S^{h}(\Omega)$. The two first terms on the right are easily bounded by

$$
h_{l}^{2-\epsilon} d_{l}^{\beta M^{-2-\epsilon}} \leqslant h^{2-\epsilon} d_{l}^{-\beta} M^{-\epsilon} .
$$

Using Theorem 4.1, a trivial bound for the last term on the right is $d_{l}^{-1} h^{2-\epsilon}$. However, employing a slightly more involved argument (involving ideas from Lemma 5.1 of Part 1, and to be given in Appendix 2), we have

$$
d_{l}^{-1}\left\|u-u_{h}\right\|_{0, \Omega} \leqslant C h^{2-\epsilon} d_{l}^{-\beta} M^{-\epsilon} .
$$

Thus, (5.2) obtains.

From (5.2) we can expect that the maximum error occurs for $d \leqslant h^{1 / \beta_{M}}$. For the stress intensity factor we obtain

$$
\left|k_{M}-k_{M}(d, h)\right| \leqslant \begin{cases}C\left(h^{1-\epsilon} d^{-\beta}+d^{\beta} M\right), & d \leqslant h^{1 / \beta} M, \\ C\left(h^{2-\epsilon} d^{-2 \beta} M^{-\epsilon}+d^{\beta}\right), & h^{1 / \beta}{ }^{1 / 3}<d<R_{M} .\end{cases}
$$

The best that can be said is now that

$$
\left|k_{M}-k_{M}\left(h^{2 / 3 \beta_{M}}, h\right)\right| \leqslant C h^{2 / 3-\epsilon} .
$$

In the two examples above, either AA. $3_{M}(r, s)$ or AA.4(r, r) (ii) $)_{M}$ took precedence in the refinement near $v_{M}$. It may of course happen that they intermix; thus, e.g. for $r=3, s=2, \beta_{M}=2 / 3$ we have the following picture:

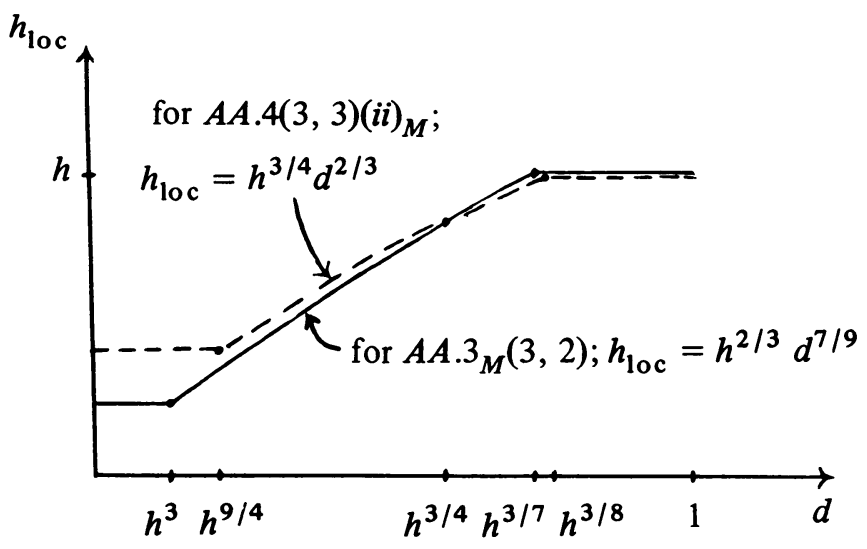

FIGURE 6

We leave the analysis of this case as an exercise for the reader. 
6. Proof of Theorem 2.1. For simplicity we assume in what follows that $R_{j}=1$, $\widetilde{R}_{j}=1 / 8$. We shall first localize the problem by way of an auxiliary mixed problem, cf. Part 1, (7.3). Let $D_{1}=\left\{x \in \bar{\Omega}:\left|x-v_{j}\right| \leqslant 1 / 4\right\}$ and $D_{2}=\left\{x \in \bar{\Omega}:\left|x-v_{j}\right| \leqslant\right.$ $1 / 2\}$ so that $\widetilde{\Omega}_{j} \oiint D_{1} \oiint D_{2} \oiint \Omega_{j}$. Let $\omega \in C^{\infty}\left(\Omega_{j}\right)$ be such that $\omega \equiv 1$ on $D_{1}$, supp $\omega \subseteq D_{2}$. Put $\tilde{u}=\omega u$, and let $\tilde{u}_{h} \in S^{h}\left(\Omega_{j}\right)$ satisfy

$$
A\left(\tilde{u}-\tilde{u}_{h}, \chi\right)=0 \text { for all } \chi \in S^{h}\left(\Omega_{j}\right)
$$

The function $\tilde{u}_{h}$ can be thought of as the approximate solution of an auxiliary mixed problem with right-hand side $-\Delta \tilde{u}$.

We have the following:

Lemma 6.1. Assume AA. $1_{j}(r)$. Given $\epsilon>0$ there exists a constant $C$ such that

$$
\left\|\tilde{u}-\tilde{u}_{h}\right\|_{L_{\infty}\left(D_{1}\right)} \leqslant C h^{r-\epsilon} .
$$

Before proving Lemma 6.1 let us show how Theorem 2.1 follows from it. We have

$$
\left\|u-u_{h}\right\|_{L_{\infty}\left(\tilde{\Omega}_{j}\right)}=\left\|\tilde{u}-u_{h}\right\|_{L_{\infty}\left(\tilde{\Omega}_{j}\right)} \leqslant\left\|\tilde{u}-\tilde{u}_{h}\right\|_{L_{\infty}\left(\tilde{\Omega}_{j}\right)}+\left\|\tilde{u}_{h}-u_{h}\right\|_{L_{\infty}\left(\tilde{\Omega}_{j}\right)}
$$

By (2.7) and (6.1), $A\left(\tilde{u}_{h}-u_{h}, \chi\right)=0$ for $\chi \in S^{h}\left(D_{1}\right)$ so that from Theorem 3.1 of Part 1 we may infer that

$$
\begin{aligned}
\left\|\tilde{u}_{h}-u_{h}\right\|_{L_{\infty}\left(\tilde{\Omega}_{j}\right)} & \leqslant C h^{-\epsilon}\left\|\tilde{u}_{h}-u_{h}\right\|_{-p, D_{1}} \\
& \leqslant C h^{-\epsilon}\left\{\left\|\tilde{u}-\tilde{u}_{h}\right\|_{L_{\infty}\left(D_{1}\right)}+\left\|u-u_{h}\right\| \|_{-p, D_{1}}\right\} .
\end{aligned}
$$

From (6.3), (6.4) and (6.2) we obtain the desired estimate (2.8).

For brevity we shall henceforth in this section write $\beta=\beta_{j}$, and $\Omega_{k}=\Omega_{j, k}$. We shall also denote

$$
d_{k}=2^{-k}, \quad d_{I}=2^{-k_{j}}, \quad d(x)=\left|x-v_{j}\right| .
$$

In the proof of Lemma 6.1 we shall need a few approximation results, all consequences of the assumption AA.2. We list them here in one place; cf. Section 1 for notation.

(i) If $1 / 2<\beta<1$, then there exists $\chi \in S^{h}\left(\Omega_{j}\right)$ such that

$$
\|\tilde{u}-\chi\|_{L_{\infty}\left(\Omega_{I}^{l}\right)}+\|\tilde{u}-\chi\|_{1, \Omega_{I}^{l}} \leqslant C h_{I}^{\beta-\epsilon} .
$$

(ii) If $1<\beta<r$, then there exists $\chi \in S^{h}\left(\Omega_{j}\right)$ such that

$$
\|\tilde{u}-\chi\|_{L_{\infty}\left(\Omega_{I}^{l}\right)}+h_{I}\|\tilde{u}-\chi\|_{w_{\infty}^{1}\left(\Omega_{I}^{l}\right)} \leqslant C h_{I}^{\beta-\epsilon} .
$$

(iii) For $1 \leqslant k \leqslant k_{j}$, there exists $\chi \in S^{h}\left(\Omega_{j}\right)$ such that

$$
\|\tilde{u}-\chi\|_{L_{\infty}\left(\Omega_{k}^{l}\right)}+h_{k}\|\tilde{u}-\chi\|_{w_{\infty}^{1}\left(\Omega_{k}^{l}\right)} \leqslant C h_{k}^{r} d_{k}^{\beta-r-\epsilon} .
$$

Here $l=0,1,2$ and the constants $C$ are independent of $h$ and, in the case of (6.8), also of $k$. 
The proofs of the above results can be accomplished as in Part 1, Lemma 4.1 (for (6.6), (6.7)) and Lemma 2.1 (for (6.8)).

We shall also need the following general approximation results:

(iv) If $1 / 2<\beta<1$, there exists a constant $C$ such that for any $v \in \dot{H}^{1}\left(\Omega_{j}\right) \cap$ $H^{1+\beta-\epsilon}\left(\Omega_{j}\right)$ there exists $\chi \in S^{h}\left(\Omega_{j}\right)$ satisfying

$$
\|v-\chi\|_{1, \Omega_{I}} \leqslant C h_{I}^{\beta-\epsilon}\|v\|_{1+\beta-\epsilon, D_{1}} .
$$

(v) There exists a constant $C$ such that for any $v \in \stackrel{<}{H}^{1}\left(\Omega_{j}\right) \cap H^{2}\left(\Omega_{j}\right)$, there exists $\chi \in S^{h}\left(\Omega_{j}\right)$ satisfying

$$
\begin{aligned}
& \|v-\chi\|_{1, \Omega_{I}} \leqslant C h_{I}\|v\|_{2, \Omega_{I}^{1}}, \\
& \|v-\chi\|_{1, \Omega_{k}} \leqslant C h_{k}\|v\|_{2, \Omega_{k}^{1}} .
\end{aligned}
$$

We point out that due to the norm on the right of (6.9) extending over all of $D_{1}$, the estimate (6.9) is not very sharp. However, it is possible to give a simple proof, following the proof of Lemma 2.2 in Part 1. In various concrete examples, the result can be sharpened. The proof of (6.10) and (6.11) can be accomplished as in Part 1, Lemma 2.1.

After these preliminaries, let us start the proof of Lemma 6.1. Let $\tilde{e}=\tilde{u}-\tilde{u}_{h}$ and $E=E(x)=\left(d(x)+d_{I}\right)^{-1} \tilde{e}$. We shall first show that given $\epsilon>0$ there exists a constant $C$ such that

$$
\|\tilde{e}\|_{L_{\infty}\left(D_{1}\right)} \leqslant C h^{-\epsilon}\left(h^{r}+\|E\|_{0, \Omega_{j}}\right)
$$

We have

$$
\|\tilde{e}\|_{L_{\infty}\left(D_{1}\right)}=\max \left(\|\tilde{e}\|_{L_{\infty}\left(\Omega_{I}\right)} \max _{k=3, \ldots, k_{j}}\|\tilde{e}\|_{L_{\infty}\left(\Omega_{k}\right)}\right)
$$

Consider first $\|\tilde{e}\|_{L_{\infty}\left(\Omega_{I}\right)}$. When $1 / 2<\beta<1$ we can apply Theorem 3.1 of Part 1; it is straightforward to verify that since $\mu>1-\beta / r$, dist $\left(\Omega_{I}, \Omega_{I}^{1}\right) \leqslant h_{I}^{1-\delta}$ for some $\delta$ $>0$ so that the theorem applies. For arbitrary $\epsilon>0$ and for any $\chi \in S^{h}$ we obtain

$$
\begin{gathered}
\|\tilde{e}\|_{L_{\infty}\left(\Omega_{I}\right)} \leqslant C h_{I}^{-\epsilon}\left\{\|\tilde{u}-\chi\|_{L_{\infty}\left(\Omega_{I}^{1}\right)}+d_{I}^{-1}\|\tilde{u}-\chi\|_{0, \Omega_{I}^{1}}\right. \\
\left.+\|\tilde{u}-\chi\|_{1, \Omega_{I}^{1}}+d_{I}^{-1}\|\tilde{e}\|_{0, \Omega_{I}^{1}}\right\} \\
\leqslant C h_{I}^{-\epsilon}\left\{\|\tilde{u}-\chi\|_{L_{\infty}\left(\Omega_{I}^{1}\right)}+\|\tilde{u}-\chi\|_{1, \Omega_{I}^{1}}+d_{I}^{-1}\|\tilde{e}\|_{0, \Omega I}\right\} ;
\end{gathered}
$$

and using (6.6) and (2.5), we clearly have

$$
\|\tilde{e}\|_{L_{\infty}\left(\Omega_{I}\right)} \leqslant C h^{-\epsilon}\left(h^{r}+\|E\|_{0, \Omega_{j}}\right), \quad 1 / 2<\beta<1 .
$$

In the case of $1<\beta<r$, we apply Theorem 3.2 of Part 1 and arrive at $\|\tilde{e}\|_{L_{\infty}\left(\Omega_{I}\right)} \leqslant C h^{-\epsilon}\left\{h_{I}\|\tilde{u}-\chi\|_{w_{\infty}^{1}\left(\Omega_{I}^{1}\right)}+\|\tilde{u}-\chi\|_{L_{\infty}\left(\Omega_{I}^{1}\right)}+d_{I}^{-1}\|\tilde{e}\|_{0, \Omega_{I}^{1}}\right\}$; 
or, by use of (6.7) and combining the result with (6.14),

$$
\|\tilde{e}\|_{L_{\infty}\left(\Omega_{I}\right)} \leqslant C h^{-\epsilon}\left\{h^{r}+\|E\|_{0, \Omega_{j}}\right\}, \quad 1 / 2<\beta<r .
$$

Next consider the error on the domains $\Omega_{k}, k=3, \ldots, k_{j}$. Using again Theorem 3.2 of Part 1 ,

$$
\|\tilde{e}\|_{L_{\infty}\left(\Omega_{k}\right)} \leqslant C h_{k}^{-\epsilon}\left\{h_{k}\|\tilde{u}-\chi\|_{w_{\infty}^{1}\left(\Omega_{k}^{1}\right)}+\|\tilde{u}-\chi\|_{L_{\infty}\left(\Omega_{k}^{1}\right)}+d_{k}^{-1}\|\tilde{e}\|_{0, \Omega_{k}^{1}}\right\} .
$$

Inserting (6.8) and using that $h^{\gamma} \leqslant h_{k} \leqslant h d_{k}^{\mu}$,

$$
\|\tilde{e}\|_{L_{\infty}\left(\Omega_{k}\right)} \leqslant C h_{k}^{-\epsilon}\left\{h_{k}^{r-\epsilon} d_{k}^{\beta-r-\epsilon}+\|E\|_{0, \Omega_{j}}\right\} \leqslant C h^{-\epsilon}\left\{h^{r}+\|E\|_{0, \Omega_{j}}\right\} .
$$

From (6.13), (6.15) and (6.16) we obtain the desired estimate (6.12).

The proof of Lemma 5.1 is now completed by using the following result in (6.12).

LeMma 6.2. Given $\epsilon>0$, there exists $a$ constant $C$ such that for $h$ sufficiently small,

$$
\|E\|_{0, \Omega_{j}} \equiv\left\|\left(d(x)+d_{I}\right)^{-1} \tilde{e}\right\|_{0, \Omega_{j}} \leqslant C h^{r-\epsilon} .
$$

In the proof of this lemma we shall need some error estimates for $\widetilde{e}$ in $H^{1}$ and $L_{2}$ in the presence of the current refinement. We have

$$
\|\widetilde{e}\|_{0, \Omega_{j}}+h\|\widetilde{e}\|_{1, \Omega_{j}} \leqslant C h^{r-\epsilon} .
$$

The proof of this fact uses much the same techniques as those employed in Appendix 1 and will therefore be left to the reader. The estimate in $H^{1}$ is immediate. To perform a duality argument, note that the solution of the mixed problem in Part 1, (7.3) with right-hand side in $L_{2}$ belongs to $H^{2-\epsilon}$ locally at the comers where the boundary conditions change, cf. [17] and [29] of Part 1.

We now start the proof of Lemma 6.2. First note that $\Omega_{j}=D_{1} \cup \Omega_{1}^{1}$, so that

$$
\|E\|_{0, \Omega_{j}} \leqslant\|E\|_{0, \Omega_{1}^{1}}+\|E\|_{0, D_{1}} .
$$

Clearly, using (6.18),

$$
\|E\|_{0, \Omega_{1}^{1}} \leqslant C\|\tilde{e}\|_{0, \Omega_{1}^{1}} \leqslant C h^{r-\epsilon} .
$$

In order to estimate $\|E\|_{0, D_{1}}$ we shall employ a "duality argument". We have

$$
\|E\|_{0, D_{1}}=\sup _{\substack{g \in C_{0}^{\infty}\left(D_{1}\right) \\\|g\|_{0, D_{1}}=1}}\left(\left(d(x)+d_{I}\right)^{-1} \tilde{e}, g\right)
$$

Let now $v$ solve the following mixed boundary value problem:

$$
\left\{\begin{aligned}
-\Delta v & =\left(d(x)+d_{I}\right)^{-1} g \quad \text { in } \Omega_{j} \\
v=0 & \text { on } \partial \Omega_{j} \cap \partial \Omega \\
\frac{\partial v}{\partial n}=0 & \text { on } \partial \Omega_{j} \backslash\left(\partial \Omega_{j} \cap \partial \Omega\right) .
\end{aligned}\right.
$$


We shall require some a priori estimates for this problem.

Leмma 6.3. Let $v$ be the solution of (6.22) where $g \in C_{0}^{\infty}\left(D_{1}\right), D_{1} \ggg \Omega_{j}$ with $\|g\|_{0}=1$. Then,

$$
\begin{gathered}
\left\{\begin{array}{l}
\|v\|_{1+\beta-\epsilon, D_{1}} \leqslant C d_{I}^{-\beta}, \quad 1 / 2<\beta<1, \\
\|v\|_{2, D_{1}} \leqslant C d_{I}^{-1-\epsilon}, \quad \beta>1,
\end{array}\right. \\
\|v\|_{2, \Omega_{k}} \leqslant C d_{k}^{-1} d_{I}^{-\epsilon}, \quad k=3, \ldots, k_{j}, \\
\|v\|_{2-\epsilon, \Omega_{1}^{1}} \leqslant C d_{I}^{-\epsilon} .
\end{gathered}
$$

The proof of this lemma will be postponed until the end of this section.

Returning now to the proof of Lemma 6.2, we have for any $\chi \in S^{h}\left(\Omega_{j}\right)$,

$$
\begin{gathered}
\left(\left(d(x)+d_{I}\right)^{-1} \tilde{e}, g\right)=A(\tilde{e}, v)=A(\tilde{e}, v-\chi) \\
\leqslant\|\tilde{e}\|_{1, \Omega_{I}}\|v-\chi\|_{1, \Omega_{I}}+\sum_{k=3}^{k_{j}}\|\tilde{e}\|_{1, \Omega_{k}}\|v-\chi\|_{1, \Omega_{k}} \\
+\|\tilde{e}\|_{1, \Omega_{1}^{1}}\|v-\chi\|_{1, \Omega_{1}^{1}}
\end{gathered}
$$

We shall estimate the terms on the right separately. $\in S^{h}$

Applying Lemma 7.2 of Part 1 to the domains $\Omega_{I}$ and $\Omega_{I}^{1}$, we have for any $\eta$

$$
\begin{aligned}
\|\tilde{e}\|_{1, \Omega_{I}} \leqslant C\left\{\|\tilde{u}-\eta\|_{1, \Omega_{I}^{1}}+d_{I}^{-1}\|\tilde{u}-\eta\|_{0, \Omega_{I}^{1}}+d_{I}^{-1}\|\tilde{e}\|_{0, \Omega_{I}^{1}}\right\} \\
\leqslant C \begin{cases}d_{I}\|\tilde{u}-\eta\|_{w_{\infty}^{1}\left(\Omega_{I}^{1}\right)}+\|\tilde{u}-\eta\|_{L_{\infty}\left(\Omega_{I}^{1}\right)}+\|E\|_{0, \Omega_{j}} & \text { for } 1<\beta, \\
\|\tilde{u}-\eta\|_{1, \Omega_{I}^{1}}+\|\tilde{u}-\eta\|_{L_{\infty}\left(\Omega_{I}^{1}\right)}+\|E\|_{0, \Omega_{j}} & \text { for } 1 / 2<\beta<1 .\end{cases}
\end{aligned}
$$

Recalling (6.6) and (6.7),

$$
\|\tilde{e}\|_{1, \Omega_{I}} \leqslant C \begin{cases}d_{I} h_{I}^{\beta-1-\epsilon}+\|E\|_{0, \Omega_{j}}, & 1<\beta, \\ h_{I}^{\beta-\epsilon}+\|E\|_{0, \Omega_{j}}, & 1 / 2<\beta<1 .\end{cases}
$$

We shall next estimate $\|\tilde{e}\|_{1, \Omega_{k}}, k=3, \ldots, k_{j}$. Again using Lemma 7.2 of Part 1, and (6.8) (noting that $\|w\|_{1, \Omega_{k}^{1}} \leqslant C d_{k}\|w\|_{W_{\infty}^{1}\left(\Omega_{k}^{1}\right)}$ ),

$$
\|\tilde{e}\|_{1, \Omega_{k}} \leqslant C\left\{h_{k}^{r-1-\epsilon} d_{k}^{\beta-r+1-\epsilon}+\|E\|_{0, \Omega_{j}}\right\} .
$$


To bound $\|\tilde{e}\|_{1, \Omega_{1}^{1}}$ we use (6.18),

$$
\|\tilde{e}\|_{1, \Omega_{1}^{1}} \leqslant C h^{r-1-\epsilon} .
$$

We shall next attack the terms involving $v-\chi$ on the right of (6.26). Using (6.9) or (6.10), and (6.23),

$$
\|v-\chi\|_{1, \Omega_{I}} \leqslant \begin{cases}C h_{I} d_{I}^{1-\epsilon}, & 1<\beta, \\ C h_{I}^{\beta-\epsilon} d_{I}^{-\beta}, & 1 / 2<\beta<1 .\end{cases}
$$

Using (6.11) and (6.24),

$$
\|v-\chi\|_{1, \Omega_{k}} \leqslant C h_{k} d_{k}^{-1-\epsilon} \text {. }
$$

Finally, utilizing Lemma 2.2 of Part 1, and (6.25),

$$
\|v-\chi\|_{1, \Omega_{1}^{1}} \leqslant C h^{1-\epsilon}\|v\|_{2-\epsilon, \Omega_{1}^{2}} \leqslant C h^{1-\epsilon} h_{I}^{-\epsilon} \text {. }
$$

Insert the results (6.27)-(6.32) into (6.26). We obtain

$$
\begin{aligned}
\left(\left(d(x)+d_{I}\right)^{-1} \tilde{e}, g\right) \leqslant & C\left\{\begin{array}{ll}
\left(d_{I} h_{I}^{\beta-1-\epsilon}+\|E\|_{0, \Omega_{j}}\right) h_{I} d_{I}^{-1-\epsilon} & \text { for } \beta>1 \\
\left(h_{I}^{\beta-\epsilon}+\|E\|_{0, \Omega_{j}}\right) h_{I}^{\beta-\epsilon} d_{I}^{-\beta} & \text { for } \beta<1
\end{array}\right\} \\
& +C \sum_{k=3}^{k_{j}}\left(h_{k}^{r-1-\epsilon} d_{k}^{\beta-r+1-\epsilon}+\|E\|_{0, \Omega_{j}}\right) h_{k} d_{k}^{-1} d_{I}^{-\epsilon} \\
& +C h^{r-1-\epsilon} h^{1-\epsilon} h_{I}^{-\epsilon} .
\end{aligned}
$$

Thus,

$$
\begin{aligned}
\left(\left(d(x)+d_{I}\right)^{-1} \tilde{e}, g\right) \leqslant & C h_{I}^{\beta-\epsilon} d_{I}^{-\epsilon}+C h_{I}^{\beta-\epsilon}\left(\frac{h_{I}^{\beta-\epsilon}}{d_{I}^{\beta}}\right) \\
& +C d_{I}^{-\epsilon} \sum_{k=3}^{k_{j}} h_{k}^{r-\epsilon} d_{k}^{\beta-r-\epsilon}+C h^{r-\epsilon} h_{I}^{-\epsilon} \\
& +C\|E\|_{0, \Omega_{j}} \times\left(\left\{\begin{array}{l}
h_{I} d_{I}^{-1-\epsilon}, \beta>1 \\
h_{I}^{\beta-\epsilon} d_{I}^{-\beta}, \beta<1
\end{array}\right\}+d_{I}^{-\epsilon} \sum_{k=3}^{k_{j}} h_{k} d_{k}^{-1}\right)
\end{aligned}
$$

Recall now from AA. ${ }_{j}(r)$ that

$$
h^{\gamma} \leqslant h_{I} \leqslant h^{r / \beta}, \quad h^{\gamma} \leqslant h_{k} \leqslant h \cdot d_{k}^{\mu}
$$

with $\mu=1-\beta / r+\delta$, for some $\delta>0$. Also, $d_{I} \geqslant h^{r / \beta-\delta^{\prime}}$, with $\delta^{\prime}>0$. Hence, from the above 


$$
\begin{aligned}
\left(\left(d(x)+d_{I}\right)^{-1} \tilde{e}, g\right) & \leqslant C h^{r-\epsilon}+C\|E\|_{0, \Omega}\left(\left\{\begin{array}{c}
h_{I}^{\delta^{\prime}-\epsilon}, \beta>1 \\
h_{I}^{\beta \delta^{\prime}-\epsilon}, \beta<1
\end{array}\right\}+d_{I}^{-\epsilon} \sum_{k=3}^{k_{j}} h d_{k}^{\mu-1}\right) \\
& \leqslant C h^{r-\epsilon}+C\|E\|_{0, \Omega}\left(\left\{\begin{array}{c}
h_{I}^{\delta^{\prime}-\epsilon}, \beta>1 \\
h_{I}^{\beta \delta^{\prime}-\epsilon}, \beta<1
\end{array}\right\}+d_{I}^{\delta-\epsilon} h^{\delta^{\prime} \beta / r}\right)
\end{aligned}
$$

The positive number $\epsilon$ can be made arbitrarily small, whereas $\delta, \delta^{\prime}>0$ are fixed.

Thus, by fixing $\epsilon$ small, hence fixing the constants $C=C_{\epsilon}$, and then taking $h$ sufficiently small, we arrive at

$$
\left(\left(d(x)+d_{I}\right)^{-1} \tilde{e}, g\right) \leqslant C h^{r-\epsilon}+1 / 2\|E\|_{0, \Omega_{j}} .
$$

Now from (6.19), (6.20) and (6.21),

$$
\|E\|_{0, \Omega_{j}} \leqslant C h^{r-\epsilon}+1 / 2\|E\|_{0, \Omega_{j}}
$$

which proves Lemma 6.2.

It remains to prove Lemma 6.3.

Proof of Lemma 6.3. To show (6.23), let $\omega \in C^{\infty}\left(\Omega_{j}\right)$ be such that $\omega \equiv 1$ on $D_{1}, \omega$ vanishes outside $D_{2}$. By use of the estimates of Part 1 , Section 1 , we find that

$$
\begin{aligned}
\|v\|_{1+\beta-\epsilon, D_{1}} \leqslant\|\omega v\|_{1+\beta-\epsilon, \Omega_{j}} \leqslant C\|\Delta(\omega v)\|_{\beta-1-\epsilon / 2, \Omega_{j}} & \left.+\|v \Delta \omega\|_{\beta-1-\epsilon / 2, D_{2}}\right\} \\
& \leqslant C\left\{\|\omega \Delta v\|_{\beta-1-\epsilon / 2, D_{2}}+\|\nabla \omega \cdot \nabla v\|_{\beta-1-\epsilon / 2, D_{2}}\right. \\
& \leqslant C\left\{\|\Delta v\|_{\beta-1-\epsilon / 2, D_{2}}+\|v\|_{1, D_{2}}\right\} \\
& \leqslant C\left\|\left(d(x)+d_{I}\right)^{-1} g\right\|_{\beta-1-\epsilon / 2, D_{1}} .
\end{aligned}
$$

Now,

$$
\text { (6.34) }\left\|\left(d(x)+d_{I}\right)^{-1} g\right\|_{\beta-1-\epsilon / 2, D_{1}}=\sup _{\psi \in H^{1-\beta+\epsilon / 2}\left(D_{1}\right)} \frac{\left(\left(d(x)+d_{I}\right)^{-1} g, \psi\right)}{\|\psi\|_{1-\beta+\epsilon / 2, D_{1}}} \text {. }
$$

Using Schwarz' inequality,

$$
\begin{aligned}
\left(\left(d(x)+d_{I}\right)^{-1} g, \psi\right) & \leqslant\left\|\left(d(x)+d_{I}\right)^{-\beta} g\right\|_{0, D_{1}}\left\|\left(d(x)+d_{I}\right)^{\beta-1} \psi\right\|_{0, D_{1}} \\
& \leqslant C d_{I}^{-\beta}\left\|\left(d(x)+d_{I}\right)^{\beta-1} \psi\right\|_{0, D_{1}} \cdot
\end{aligned}
$$

By Hölder's inequality, and Sobolev's inequality (cf. Part 1, (1.6)), with $q=$ $(8+\beta \epsilon) /(8-4 \beta+\beta \epsilon), q^{\prime}=2 / \beta+\epsilon / 4$, 


$$
\begin{aligned}
\left\|\left(d(x)+d_{I}\right)^{\beta-1} \psi\right\|_{0, D_{1}} & \leqslant\left\|\left(d(x)+d_{I}\right)^{\beta-1}\right\|_{L_{q}\left(D_{1}\right)}\|\psi\|_{L_{q^{\prime}}\left(D_{1}\right)} \\
& \leqslant C\|\psi\|_{1-\beta+\epsilon / 2, D_{1}}
\end{aligned}
$$

From this and (6.33), (6.34) we obtain (6.23).

To show (6.24), we have, cf. Part 1, Lemmas 8.2 and 8.3,

$$
\|v\|_{2, \Omega_{k}^{1}} \leqslant C\left\{\|\Delta v\|_{0, \Omega_{k}^{2}}+d_{k}^{-1}\|v\|_{1, \Omega_{k}^{2}}\right\} .
$$

As in the proof of Lemma 8.3, Part 1, we see that

$$
\|v\|_{1, \Omega_{j}} \leqslant C d_{I}^{-\epsilon}
$$

and thus,

$$
\|v\|_{2, \Omega, \frac{1}{k}} \leqslant C\left\{\left\|\left(d(x)+d_{I}\right)^{-1} g\right\|_{0, \Omega_{k}^{2}}+d_{k}^{-1} d_{I}^{-\epsilon}\right\}
$$

which proves (6.24).

The inequality (6.25) follows from the fact that the problem (6.22) has local $H^{2-\epsilon}$ regularity at the right angled corners where the boundary conditions change. By the support properties of $g, v$ is harmonic on $\Omega_{1}^{1}$ and one obtains

$$
\|v\|_{2-\epsilon, \Omega_{1}^{1}} \leqslant C\|v\|_{1, \Omega_{1}^{2}}
$$

An application of (6.35) now verifies (6.25).

This proves Lemma 6.3.

The proof of Theorem 2.1 is now complete.

Appendix 1. Proof of Theorem 4.1. As set forth in Section 4, it suffices to prove that in the presence of condition AA.4 $(r, s)$, we have for any $g \in H^{r-2}(\Omega)$

where

$$
\inf _{\chi \in S^{h}(\Omega)}\|v-\chi\|_{1, \Omega} \leqslant C h^{s / 2-\epsilon}\|g\|_{r-2, \Omega}
$$

$$
-\Delta v=g \text { in } \Omega, \quad v=0 \text { on } \partial \Omega .
$$

It is a consequence of our assumptions that there exists a $\chi \in S^{h}(\Omega)$ such that

$$
\|v-\chi\|_{1, \Omega_{j, I}} \leqslant C h_{j, I}^{\min \left(\beta_{j}, r-1\right)-\epsilon}\|v\|_{\min \left(1+\beta_{j}, r\right)-\epsilon, \Omega_{j}},
$$

$$
\|v-\chi\|_{1, \Omega_{0}} \leqslant C h^{r-1}\|v\|_{r, \Omega_{0}^{\prime}}
$$

$$
\|v-\chi\|_{1, \Omega_{j, k}} \leqslant C h_{j, k}^{r-1}\|v\|_{r, \Omega_{j, k}^{\prime}}
$$

for $j=1, \ldots, M, k=1, \ldots, k_{j}\left(h_{j, k}=h\right.$ in case $\left.k<k_{0, j}\right)$. Here $\Omega_{0} \ggg \Omega_{0}^{\prime} \ggg \Omega$. For the above, cf. (6.9)-(6.11).

We shall use the following local a priori estimates for the problem (A.1.2).

LEMMA A.1.1.

$$
\|v\|_{\min \left(1+\beta_{j}, r\right)-\epsilon, \Omega_{j}} \leqslant C\|g\|_{r-2, \Omega},
$$


(A.1.7)

$$
\|v\|_{r, \Omega 1}^{1, k} \leqslant C d_{k}^{-r+1+\min \left(\beta_{j}, r-1\right)-\epsilon}\|g\|_{r-2, \Omega}, \quad\left(d_{k}=2^{-k}\right)
$$

Before proving this lemma, let us show how (A.1.1) follows from it. We have

$$
\|v-\chi\|_{1, \Omega} \leqslant \sum_{j=1}^{M}\|v-\chi\|_{1, \Omega}+\|v-\chi\|_{1, \Omega_{0}} .
$$

Consider a fixed $j, 1 \leqslant j \leqslant M$. The other corners, and the interior domain $\Omega_{0}$ are treated similarly.

We write

$$
\|v-\chi\|_{1, \Omega_{j}} \leqslant\|v-\chi\|_{1, \Omega_{j, I}}+\sum_{k=1}^{k_{j}}\|v-\chi\|_{1, \Omega},
$$

and using (A.1.3) and (A.1.5), and then Lemma A.1.1,

$$
\|v-\chi\|_{1, \Omega_{j}} \leqslant C h_{I}^{\min \left(\beta_{j}, r-1\right)-\epsilon}\|v\|_{\min \left(1+\beta_{j}, r\right)-\epsilon, \Omega_{j}}+\sum_{k=1}^{k_{j}} h_{j, k}^{r-1}\|v\|_{r, \Omega_{j, k}^{1}}
$$

$$
\leqslant C\left[h_{I}^{\min \left(\beta_{j}, r-1\right)-\epsilon}+\sum_{k=1}^{k_{j}} h_{j, k}^{r-1} d_{k}^{-r+1+\min \left(\beta_{j}, r-1\right)-\epsilon}\right]\|g\|_{r-2, \Omega} .
$$

Consider now the quantity in square brackets. If $\beta_{j} \geqslant s / 2$, then the local meshsizes are all comparable to $h$; and since $r-1 \geqslant s / 2$ and $d_{I} \geqslant h$, we obtain

$$
\|v-\chi\|_{1, \Omega_{j}} \leqslant C\left[h^{\min \left(\beta_{j}, r-1\right)-\epsilon}\right.
$$

$$
\begin{aligned}
& \left.\quad+h^{r-1} \max \left(d_{I}^{-r+1+\min \left(\beta_{j}, r-1\right)-\epsilon}, 1\right)\right]\|g\|_{r-2, \Omega} \\
& \leqslant C h^{s / 2-\epsilon}\|g\|_{r-2, \Omega}, \quad \beta_{j} \geqslant s / 2 .
\end{aligned}
$$

In the case that $\beta_{j}<s / 2$, we use the conditions on $h_{j, k}$ set forth in $\mathrm{AA.4}(r, s)$; and since $\bar{\mu}_{j} \geqslant 1-\beta_{j} /(r-1), d_{I} \geqslant h_{I} \geqslant h^{\gamma}$, we obtain for $\epsilon$ small

(A.1.11)

$$
\begin{aligned}
& \|v-\chi\|_{1, \Omega} \\
& \leqslant C\left[h^{s\left(\beta_{j}-\epsilon\right) / 2 \beta_{j}}+\sum_{k=1}^{k_{j}} h^{s(r-1) / 2(r-1)} d_{k}^{\bar{\mu}_{j}(r-1)-r+1+\beta_{j}-\epsilon}\right]\|g\|_{r-2, \Omega} \\
& \leqslant C h^{s / 2-\epsilon}\left[1+\sum_{k=1}^{k_{j}} d_{k}^{-\epsilon}\right]\|g\|_{r-2, \Omega} \\
& \quad \leqslant C h^{s / 2-\epsilon}\left[1+d_{I}^{-\epsilon}\right]\|g\|_{r-2, \Omega} \leqslant C h^{s / 2-\epsilon}\|g\|_{r-2, \Omega}, \quad \beta_{j}<\frac{s}{2} .
\end{aligned}
$$

Combining (A.1.10) and (A.1.11) for all $j$, and with the easy estimate for $\|v-\chi\|_{1, \Omega_{0}}$ we have verified (A.1.1).

It remains to show Lemma A.1.1. 
Proof of Lemma A.1.1. The estimate (A.1.6) follows from (1.7) of Part 1 by a simple localization argument.

For (A.1.7), let us give the details in the case of $\beta_{j}<1$. Let $\omega_{1}, \omega_{2}, \omega_{3} \in$ $C^{\infty}\left(\Omega_{j, k}^{2}\right)$ and such that $\omega_{1}+\omega_{2}+\omega_{3} \equiv 1$ on $\Omega_{j, k}^{1},\left\|\omega_{l}\right\|_{W_{\infty}}^{i} \leqslant C d_{k}^{-i}, i=0,1,2, \ldots$, and their supports are as indicated in the following figure:

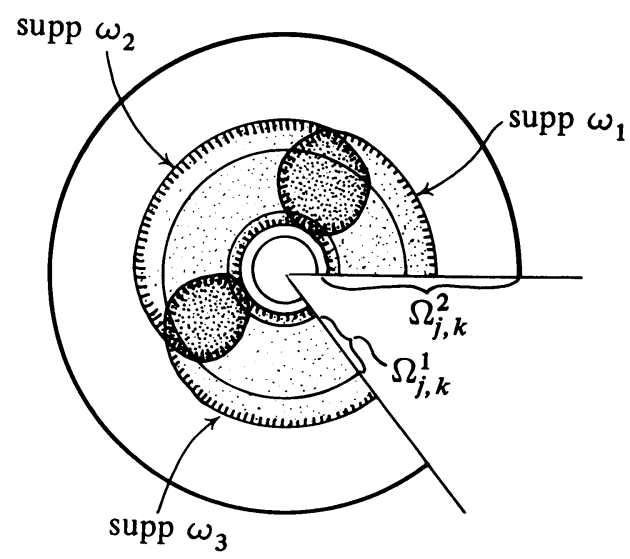

Figure 7

Then,

$$
\|v\|_{r, \Omega \underset{j, k}{1}} \leqslant \sum_{l=1}^{3}\left\|\omega_{l} v\right\|_{r, \Omega i j, k}^{1}
$$

Consider e.g. $\omega_{1} v$. By considering a suitably localized halfplane problem, we have

$$
\left\|\omega_{1} v\right\|_{r, \Omega i}^{1} \leqslant C\left\|\Delta\left(\omega_{1} v\right)\right\|_{r-2, \Omega}
$$

with $C$ independent of $k$. Now,

$$
\left\|\Delta\left(\omega_{1} v\right)\right\|_{r-2, \Omega} \leqslant C\left\{\|g\|_{r-2, \Omega_{k}^{2}}+d_{k}^{-1}\|v\|_{r-1, \Omega_{k}^{2}}+d_{k}^{-2}\|v\|_{r-2, \Omega_{k}^{2}}\right\} .
$$

For a term like $d_{k}^{-1}\|v\|_{r-1, \Omega_{k}^{2}}$ the same procedure yields

$$
d_{k}^{-1}\|v\|_{r-1, \Omega_{k}^{2}} \leqslant C\left\{d_{k}^{-1}\|g\|_{r-3, \Omega_{k}^{3}}+d_{k}^{-2}\|v\|_{r-2, \Omega_{k}^{3}}+d_{k}^{-3}\|v\|_{r-3, \Omega_{k}^{3}}\right\}
$$

and eventually, we obtain with $\Omega_{j, k} \Varangle \Omega_{j, k}^{\prime}$, diam $\Omega_{j, k}^{\prime} \leqslant C d_{k}$,

$$
\|v\|_{r, \Omega, 1}^{1, k} \leqslant C\left(\sum_{m=0}^{r-2} d_{k}^{-m}\|g\|_{r-2-m, \Omega_{j, k}^{\prime}}+d_{k}^{-r+1}\|v\|_{1, \Omega_{j, k}^{\prime}}+d_{k}^{-r}\|v\|_{0, \Omega_{j, k}^{\prime}}\right) \text {. }
$$

Let us next consider, e.g., the term $\|v\|_{1, \Omega_{j, k}^{\prime}}$. By Hölder's inequality,

$$
\|v\|_{1, \Omega_{j, k}^{\prime}} \leqslant C d_{k}^{1 / p^{\prime}}\|v\|_{w_{2 p}^{1}\left(\Omega_{j}\right)}
$$

and choosing (in the case $\left.\beta_{j}<1\right), p<1 /\left(1-\beta_{j}\right.$ ) but close; and hence $p^{\prime} \simeq 1 / \beta_{j}$, we have by Sobolev's inequality (Part 1, (1.6)) and (A.1.6)

$$
\|v\|_{1, \Omega_{j, k}^{\prime}} \leqslant C d_{k}^{\beta_{j}-\epsilon}\|v\|_{1+\beta_{j}-\epsilon / 2, \Omega_{j}} \leqslant C d_{k}^{\beta_{j}-\epsilon}\|g\|_{\beta_{j}-1-\epsilon, \Omega} .
$$


By much the same procedure,

and

$$
\|v\|_{0, \Omega_{j, k}^{\prime}} \leqslant C d_{k}^{1+\beta_{j}-\epsilon}\|g\|_{\beta_{j}-1-\epsilon, \Omega}
$$

$$
\|g\|_{r-2-m, \Omega_{j, k}^{\prime}} \leqslant C d_{k}^{m-\epsilon}\|g\|_{r-2, \Omega} .
$$

Thus, since $\beta_{j}-1<r-2$,

$$
\|v\|_{r, \Omega 1}^{1, k} \leqslant C d_{k}^{-r+1+\beta_{j}-\epsilon}\|g\|_{r-2, \Omega}, \quad \beta_{j}<1 .
$$

The case of $\beta_{j}>1$ is handled similarly.

This proves Lemma A.1.1 and completes the proof of Theorem 4.1.

Appendix 2. Proof of (5.3) in Example 5.2. We have

Fixing $g$, let $v$ be the solution of

$$
\left\|u-u_{h}\right\|_{0, \Omega \frac{1}{M, l}}=\sup _{g \in C_{0}^{\infty}\left(\Omega_{M, l}^{1}\right)} \frac{\left(u-u_{h}, g\right)}{\|g\|_{0, \Omega}}
$$

$$
\begin{cases}-\Delta v=g & \text { in } \Omega(g \text { extended by zero }) \\ v=0 & \text { on } \partial \Omega .\end{cases}
$$

For any $\chi \in S^{h}(\Omega)$ we have

$$
\left|\left(u-u_{h}, g\right)\right|=\left|A\left(u-u_{h}, v-\chi\right)\right| \leqslant\left\|u-u_{h}\right\|_{1, \Omega}\|v-\chi\|_{1, \Omega} .
$$

By (A.1.1) we have in this case (AA.4 (2,2) holds),

$$
\left\|u-u_{h}\right\|_{1, \Omega} \leqslant C \inf _{\psi \in S^{h}(\Omega)}\|u-\psi\|_{1, \Omega} \leqslant C h^{1-\epsilon} .
$$

Thus, in order to show (5.1), it remains to prove that with suitable $\chi \in S^{h}(\Omega)$,

(A.2.2) $\|v-\chi\|_{1, \Omega} \leqslant C h^{1-\epsilon} d_{M, l}^{1-\beta} M^{-\epsilon}\|g\|_{0, \Omega}, \quad g \in C_{0}^{\infty}\left(\Omega_{M, l}^{1}\right), d_{M, l} \geqslant h^{1 / \beta} M$, where $v$ and $g$ are connected by (A.2.1).

The proof will more or less consist of repeating arguments from Appendix 1, however, in a more careful way at certain crucial steps and also using the fact that $g$ has small support. For the approximation theory needed, see (A.1.3)-(A.1.5).

Write

$$
\begin{aligned}
\|v-\chi\|_{1, \Omega} \leqslant & \sum_{j=1}^{M-1}\left(\|v-\chi\|_{1, \Omega_{j, I}}+\sum_{k=1}^{k_{j}}\|v-\chi\|_{1, \Omega_{j, k}}\right) \\
& +\|v-\chi\|_{1, \Omega_{M, I}}+\sum_{k=1}^{k_{M}}\|v-\chi\|_{1, \Omega_{M, k}}+\|v-\chi\|_{1, \Omega_{0}}
\end{aligned}
$$

Consider first the terms $\|v-\chi\|_{1, \Omega_{j, P}} j=1, \ldots, M-1$. We have (assuming 
$\left.\beta_{j}<1\right)$

$$
\|v-\chi\|_{1, \Omega_{j, I}} \leqslant C h_{j, I}^{\beta_{j}-\epsilon}\|v\|_{1+\beta_{j}-\epsilon, \Omega_{j}}
$$

Since $v$ is harmonic on $\Omega_{j}$, we infer by a localization argument, (cf. Part $1,(1.7)$ ),

$$
\|v\|_{1+\beta_{j}-\epsilon, \Omega_{j}} \leqslant C\|v\|_{1, \Omega} \leqslant C\|g\|_{\beta_{M^{-1-\epsilon, \Omega}}} .
$$

Now use the fact that (see the last part of the proof of Lemma 5.1 in Part 1),

$$
\|g\|_{\beta_{M^{-1-\epsilon, \Omega}}} \leqslant C d_{M, l}^{1-\beta_{M}-\epsilon}\|g\|_{0, \Omega}, \quad g \in C_{0}^{\infty}\left(\Omega_{M, l}^{1}\right) \text {. }
$$

Thus, using AA.4(2.2) (ii) ${ }_{j}$,

$$
\|v-\chi\|_{1, \Omega_{j, I}} \leqslant C\left(h^{1 / \beta_{j}}\right)^{\beta_{j}-\epsilon} d_{M, l}^{1-\beta_{M}-\epsilon}\|g\|_{0, \Omega}
$$

$$
\leqslant C h^{1-\epsilon} d_{M, l}^{1-\beta} M^{-\epsilon}\|g\|_{0, \Omega}, \quad j=1, \ldots, M-1 .
$$

The same estimate is easily derived also for $\beta_{j}>1$, replacing the right-hand side of (A.2.4) by $C h\|v\|_{2, \Omega_{j}}$ and continuing as above.

Next, consider $\|v-\chi\|_{1, \Omega_{j, k}}, j=1, \ldots, M-1$. Now,

$$
\|v-\chi\|_{1, \Omega_{j, k}} \leqslant C h_{j, k}\|v\|_{2, \Omega, j, k} .
$$

We use the fact that since $v$ is harmonic,

$$
\|v\|_{2, \Omega, 1} \leqslant C d_{j, k}^{-1+\beta_{j}-\epsilon}\|v\|_{1+\beta,-\epsilon, \Omega_{j}}, \quad \beta_{j}<1 .
$$

This follows, e.g., by first using Lemma 8.3 of Part 1, obtaining

and then the fact that

$$
\|v\|_{2, \Omega, k} \leqslant C d_{j, k}^{-1}\|v\|_{1, \Omega_{j, k}^{2}},
$$

$$
\|v\|_{1, \Omega_{j, k}^{2}} \leqslant C d_{j, k}^{\beta_{j}-\epsilon}\|v\|_{1+\beta_{j}-\epsilon, \Omega_{j}}
$$

which is proved by applying the same techniques as in Lemma 5.1 of Part 1.

Thus, combining the above with (A.2.5) and (A.2.6), and using AA.4(2,2),

$$
\begin{aligned}
\|v-\chi\|_{1, \Omega} & \leqslant C h_{j, k} d_{j, k}^{-1+\beta_{j}-\epsilon} d_{M, l}^{1-\beta_{M}-\epsilon}\|g\|_{0, \Omega} \\
& \leqslant C h^{1-\epsilon} d_{M, l}^{1-\beta_{M}-\epsilon}\|g\|_{0, \Omega}, \quad \beta_{j}<1 .
\end{aligned}
$$

Again, the same estimate can be deduced also for $\beta_{j}>1$.

The estimates (A.2.7) and (A.2.9) can also be deduced, by the same procedure, on the domains $\Omega_{M, I}$ and $\Omega_{M, k}, k=1, \ldots, l-2, l+2, \ldots, k_{M}$, since $v$ is harmonic on $\Omega_{M, k}^{2}$. To estimate, e.g., $\|v-\chi\|_{1, \Omega_{M, l}}$ itself, we have

$$
\|v-\chi\|_{1, \Omega} \leqslant C h_{M, l}\|v\|_{2, \Omega}^{1}, ;
$$

and we then use the fact (corresponding to (A.2.8), cf. also the proof of Lemma A.1.1, and (1.7) of Part 1, and (A.2.6))

$$
\|v\|_{2, \Omega 1}^{1} \leqslant C\left(\|g\|_{0, \Omega}+d_{M, l}^{-1+\beta_{M}-\epsilon}\|v\|_{1+\beta_{M}-\epsilon, \Omega}\right) \leqslant C d_{M, l}^{-\epsilon}\|g\|_{0, \Omega}
$$


Thus, by AA.4(2,2) (ii) $)_{M}$, which is the dominant refinement around $v_{M}$, cf. Figure 5,

$$
\|v-\chi\|_{1, \Omega}, \tilde{\imath} \leqslant C h_{M, \tilde{\imath}} d_{M, \tilde{l}}^{-1}\|g\|_{0, \Omega}
$$

$$
\leqslant C h^{1-\epsilon} d_{M, \tilde{l}^{1-\epsilon}}^{-\beta_{M}}\|g\|_{0, \Omega}, \quad \tau=l-1, l, l+1 .
$$

One deduces also, easily, that

$$
\|v-\chi\|_{1, \Omega_{0}} \leqslant C h^{1-\epsilon} d_{M, l}^{1-\beta_{M}-\epsilon}\|g\|_{0, \Omega}
$$

Inserting (A.2.7) (which held also for $j=M$ ), (A.2.9) (valid also for $j=M, k=1$, $\left.\ldots, l-2, l+2, \ldots, k_{M}\right)$ and (A.2.10), (A.2.11) into (A.2.3), we obtain the desired estimate (A.2.2).

This completes the proof of (5.3).

\section{Mathematics Department \\ Cornell University \\ Ithaca, New York 14853}

1. J. R. RICE, "On the degree of convergence of nonlinear spline approximation," Approximations with Special Emphasis on Spline Functions, I. J. Schoenberg, (Ed.), Academic Press, New York, 1969, pp. 349-365.

2. A. H. SCHATZ \& L. B. WAHLBIN, "Maximum norm estimates in the finite element method on plane polygonal domains. Part 1," Math. Comp., v. 32, 1978, pp. 73-109. 